\title{
Uniform energy and density distribution: diblock copolymers' functional
}

\author{
EMANUELE NUNZIO SPADARO \\ Institut für Mathematik, Universität Zürich \\ E-mail: emanuele.spadaro@math.uzh.ch
}

[Received 15 July 2008 and in revised form 20 January 2009]

\begin{abstract}
We study a nonlocal variational problem arising in diblock copolymers models, whose energy is given by the Cahn-Hilliard functional plus a long-range interaction term. We prove that minimizers develop uniform energy and density distributions, thus justifying partially the highly regular microphase separation observed in diblock copolymers' melts. We also give a new proof of the scaling law for the minimum energy. This work extends the techniques introduced in [1] where analogous results are proved for the sharp interface limit of the functional considered.
\end{abstract}

\section{Introduction}

We study the following nonlocal perturbation of the Cahn-Hilliard energy, introduced by Ohta and Kawasaki in [28] in order to model diblock copolymers' melts:

$$
F_{\varepsilon, \sigma}(u):=\int_{\Omega}\left(\varepsilon^{2}|\nabla u(x)|^{2}+W(u(x))+\sigma\left|(-\Delta)^{-1 / 2}(u(x)-m)\right|^{2}\right) \mathrm{d} x .
$$

A diblock copolymer molecule is a linear chain consisting of two subchains (made of two different monomers, say $A$ and $B$ ) joined covalently to each other. The phenomenon Ohta and Kawasaki were interested in is the formation of highly regular patterns in diblock copolymers' melts. In solution, indeed, the two monomers repel each other; but these forces, even causing a segregation between the two subchains, cannot detach them, because of the chemical bond. The result of this competition is a microphase separation, that is, the appearance on a mesoscopic scale of highly regular structures of rich $A$ and $B$ domains (for example lamellars, bcc centered spheres, circular tubes or bycontinuous gyroids, see for example [4] and [19]) which make diblock copolymers of great interest in material science.

In the Ohta-Kawasaki functional $F_{\varepsilon, \sigma}, u$ is the density parameter describing the system: it is the difference between the averaged densities of monomers $A$ and $B$, so it takes values in $[-1,1]$ and $u= \pm 1$ when, roughly speaking, there is concentration of a single monomer. The total average of $u$ is $m$ and $W$ is a continuous positive double-well potential, which is zero only in the pure states \pm 1 . The parameters $\varepsilon$ and $\sigma$ are related to the physical properties of the melt (see [11] for details): $\varepsilon$ is proportional to the thickness of the transition regions between the two monomers, while $\sigma$ is inversely proportional to the square of the number of monomers per molecule.

As explained in [11] and [26], the most appropriate regime to model the microphase separation is given by

$$
0<\varepsilon \ll \sigma \ll 1 .
$$

From the mathematical point of view, this regime gives rise to significantly new phenomena with respect to the Cahn-Hilliard functional. 
In this work we analyze the minima of the Ohta-Kawasaki functional $F_{\varepsilon, \sigma}$ under the regime (1.2), giving a partial mathematical rigorous explanation to the regular pattern formation in diblock copolymers' melt. We prove that "statistical" quantities, such as energy and density, are uniformly distributed on a scale determined by the parameters of the functional. More precisely, we show that, in subdomains of size of order $(\varepsilon / \sigma)^{1 / 3}$, minimizers have energy approximately constant and $u$ has average approximately $m$ (which is the average on the entire domain). Moreover, we prove that the minimum energy scales as $\varepsilon^{2 / 3} \sigma^{1 / 3}$, improving Choksi's result in [8], where this scaling is proved under more restrictive hypotheses. We notice that both these scales, the one of the minimum energy and that of the characteristic size of the patterns, agree with experiments and numerical simulations.

These results are the diffusive interface counterpart of those proved in [1] by Alberti, Choksi and Otto for the sharp interface limit of the Ohta-Kawasaki functional (in the sense of $\Gamma$-convergence),

$$
\int_{\Omega}\left(|\nabla u(x)|+\left|(-\Delta)^{-1 / 2}(u(x)-m)\right|^{2}\right) \mathrm{d} x,
$$

defined for functions $u \in B V(\Omega ; \pm 1)$.

The present work is, indeed, based on the approach and techniques developed in [1]. Its new contribution is twofold: on one hand, it extends the result of uniform energy and density distribution to the more physically significant case of diblock copolymers' functional $F_{\varepsilon, \sigma}$ (which, incidentally, was one of the main motivations for [1]). And, on the other hand, despite the technical difficulties coming from the parameters $\varepsilon$ and $\sigma$, in this paper we simplify the proof of the main estimate in [1], providing a new energy bound for the nonlocal term in Lemma 4.1, this bound, although weaker than the original one, has a much clearer and direct proof and it suffices to make the main argument work.

Apart from the underlying physical model, the Ohta-Kawasaki functional has its own mathematical interest because it gives one of the simplest examples of energy-driven pattern formation arising from the competition between short-range and long-range interactions. This kind of phenomena has been observed in many variational problems, as, for example, the ones related to the study of magnetic domains (cf. [20] and the references therein), and has got much attention in the last years.

Nevertheless, it is worth stressing that, as explained in [1], the uniform distribution results proved here are not strong enough to deduce that minimizers are periodic or nearly periodic, since even highly nonperiodic structures could have the same property.

In the last years there has been an intensive study of the Ohta-Kawasaki functional. A forerunner of this interest was the work by S. Müller [24], who proved that minimizers of the one-dimensional functional

$$
H_{\varepsilon}(v):=\int_{0}^{1}\left[\varepsilon^{2} \ddot{v}(x)^{2}+W(\dot{v}(x))+v(x)^{2}\right] \mathrm{d} x
$$

are periodic. Müller introduced this functional in the context of coherent solid-solid phase transitions, but it is easily recognized, setting $u=\dot{v}$, that $H_{\varepsilon}$ reduces to the Ohta-Kawasaki functional in the case $m=0$ and $\sigma=1$.

After that work, there have been other proofs of this one-dimensional result under general hypotheses (in particular removing the symmetry intrinsic in the assumption $m=0$ ): we refer, for example, to the recent works of X. Ren and J. Wei [29], [31], X. Chen and Y. Oshita [6], and Yip [43], who proved that minimizers of the 1-d Ohta-Kawasaki functional are periodic with a period of order $(\varepsilon / \sigma)^{1 / 3}$ and have minimum energy scaling as $\varepsilon^{2 / 3} \sigma^{1 / 3}$. 
On the other hand, not much has been proved in higher dimensions without making a priori assumptions on minimizers' structure, as done, for example, in the works of Ren and Wei [33][37]. Apart from the study of the sharp interface limit made by Alberti, Choksi and Otto [1], to our knowledge, the only result obtained under mild hypotheses is the scaling law for minimizers provided by Choksi [8]. Nevertheless, there exists a wide experimental literature (see, for example, [4], [42]) and numerical simulations (see, for example, [3], [41]) suggesting that in higher dimensions minimizers are also nearly periodic. This conjecture, however, seems to be much more challenging than in 1-d. The reason is that, in the latter case, the functional is in fact local (as is clear from (1.4p) and, on the other hand, it allows the use of ODE techniques which are no more available in higher dimensions.

The paper is organized as follows. In Section 2 we fix the notations and present the results. We give three different formulations of the uniform energy distribution, this allowing us to simplify its proof, given in Section 3. The main argument in it is an accurate estimate of the nonlocal part of the energy, whose proof is postponed to Section 4 Finally, in Section 5 , via $\Gamma$-convergence, we establish a connection between the Ohta-Kawasaki functional and its sharp interface limit and prove the scaling law of the minimum energy.

This work is part of the author's master thesis [40].

\section{Notations and results}

In this section we fix the notations and state the results we are going to prove. First of all, we specify all the terms in the Ohta-Kawasaki functional $F_{\varepsilon, \sigma}$.

For simplicity, in order to minimize the influence of the domain geometry, we let $\Omega$ be the unit cube,

$$
\Omega:=Q_{1}:=(-1 / 2,1 / 2)^{n} \subset \mathbb{R}^{n},
$$

on which we fix periodic boundary conditions. In other words, we consider $\Omega:=\mathbb{T}_{1}$, the $n$ dimensional torus obtained by identifying opposite faces of the unit cube.

We search the minimum of $F_{\varepsilon, \sigma}$ in the class of Sobolev functions with periodic traces and average $m$,

$$
u \in W^{1,2}\left(\mathbb{T}_{1} ;[-1,1]\right) \quad \text { and } \quad \int_{Q_{1}} u=m \in(-1,1) .
$$

As explained in the Introduction, in the model $u$ represents the difference between the averaged densities of the two different monomers, hence it has values in $[-1,1]$.

Moreover, $\Delta$ denotes the Laplace operator with periodic boundary conditions and $W$ is a continuous positive function with zeros only at \pm 1 .

We fix the notation $Q_{l}(x)$ for the cube centered at $x$ with side length $l$,

$$
Q_{l}(x):=(-l / 2, l / 2)^{n}+x,
$$

and $\mathbb{T}_{l}$ for the $n$-dimensional torus obtained by identifying opposite faces of $Q_{l}$. Moreover, every time we consider the Poisson equation in a torus, written as

$$
\Delta u=f \quad \text { in } \mathbb{T}_{l},
$$

we mean it is satisfied with periodic boundary conditions. 
REMARK 2.1 With minor technical modifications, all the results of the paper can be proved for homogeneous Neumann boundary conditions. We choose periodic boundary condition just for the sake of simplicity: indeed, in the regime of small $\varepsilon$ and $\sigma$ and sufficiently far from the boundary, the results do not seem to be influenced by either the shape of the domain or the boundary conditions. The advantage of the periodic setting is to avoid all the complications coming from the geometry of the domain.

In order to prove uniform energy distribution, our energy being nonlocal, we have to specify what we mean by "energy in a subdomain". We start by writing the nonlocal term in the following way:

$$
\begin{aligned}
\int_{\mathbb{T}_{1}}\left|(-\Delta)^{-1 / 2}(u-m)\right|^{2} & =\int_{\mathbb{T}_{1}}\left((-\Delta)^{-1}(u-m)\right)(u-m)=\|u-m\|_{H^{-1}\left(\mathbb{T}_{1}\right)}^{2} \\
& =\int_{\mathbb{T}_{1}}|\nabla v|^{2}
\end{aligned}
$$

where $v$ solves

$$
-\Delta v=u-m \quad \text { in } \mathbb{T}_{1} .
$$

Then, for any subdomain $D \subseteq \mathbb{T}_{1}$, we set

$$
F_{\varepsilon, \sigma}(u, D):=\int_{D}\left(\varepsilon^{2}|\nabla u|^{2}+W(u)+\sigma|\nabla v|^{2}\right) .
$$

The main result asserts that, in subcubes of $\mathbb{T}_{1}$ of size $(\varepsilon / \sigma)^{1 / 3}$, the energy of a minimizer is approximately equal (up to a constant factor depending on $\varepsilon$ and $\sigma$ but independent of the subcube) to $\varepsilon^{2 / 3} \sigma^{1 / 3}$ times the volume of the subdomain.

THEOREM 1 There exist positive constants $\varrho_{0}, \gamma_{\varepsilon, \sigma}$ and $C$ such that, for every $\varepsilon / \sigma \leqslant \varrho_{0}, \varrho_{0}^{-1 / 3} \leqslant$ $l \leqslant(\sigma / \varepsilon)^{1 / 3}$ and $x \in \mathbb{T}_{1}$, each minimizer $u_{\varepsilon, \sigma}$ of $F_{\varepsilon, \sigma}$ in $\mathbb{T}_{1}$ satisfies

$$
\left|\frac{F_{\varepsilon, \sigma}\left(u_{\varepsilon, \sigma}, Q_{(\varepsilon / \sigma)^{1 / 3} l}(x)\right)}{\varepsilon^{2 / 3} \sigma^{1 / 3}\left((\varepsilon / \sigma)^{1 / 3} l\right)^{n}}-\gamma_{\varepsilon, \sigma}\right| \leqslant C\left(\frac{1}{l}+\varepsilon^{2 / 3} \sigma^{1 / 3}\right) .
$$

A byproduct of Theorem 1 is the proof of the uniform density distribution, which asserts that the average of $u$ over subcubes of order $(\varepsilon / \sigma)^{1 / 3}$ is approximately $m$.

Proposition 2.1 There exist positive constants $\varrho_{0}$ and $C$ such that, for every $\varepsilon / \sigma \leqslant \varrho_{0}, \varrho_{0}^{-1 / 3} \leqslant$ $l \leqslant(\sigma / \varepsilon)^{1 / 3}$ and $x \in \mathbb{T}_{1}$, each minimizer $u_{\varepsilon, \sigma}$ of $F_{\varepsilon, \sigma}$ satisfies

$$
\left|f_{Q_{(\varepsilon / \sigma)^{1 / 3} l}(x)} u_{\varepsilon, \sigma}-m\right| \leqslant \frac{C}{l} \text {. }
$$

Notice that Theorem 1 alone does not imply that the energy in subdomains scales as $\varepsilon^{2 / 3} \sigma^{1 / 3}$ : to deduce this, we have to prove a uniform bound for the $\gamma_{\varepsilon, \sigma}$ 's. Actually, we prove more, showing that these constants have a limit as $\varepsilon / \sigma \rightarrow 0$.

Proposition 2.2 There exists a constant $\gamma>0$ such that

$$
\lim _{\varepsilon / \sigma \rightarrow 0} \gamma_{\varepsilon, \sigma}=\gamma
$$




\section{$2.1 \quad$ Rescaled variables}

The starting point of our analysis is the following rescaling of factor $\varrho=(\varepsilon / \sigma)^{1 / 3}$ :

$$
w(y)=u(\varrho y) \quad \text { for } y \in \mathbb{T}_{\varrho^{-1}} .
$$

Writing for brevity $\epsilon=\varepsilon^{2 / 3} \sigma^{1 / 3}$, in these new variables, the functional assumes the form

$$
F_{\varepsilon, \sigma}(u, \Omega)=\frac{\epsilon}{\left|\Omega_{\varrho^{-1}}\right|} \int_{\Omega_{\varrho^{-1}}}\left(\epsilon|\nabla w|^{2}+\epsilon^{-1} W(w)+|\nabla \zeta|^{2}\right),
$$

where $\zeta$ solves

$$
-\Delta \zeta=w-m \quad \text { in } \mathbb{T}_{\varrho^{-1}}
$$

Defining

$$
M_{\epsilon}(u, D):=\int_{D}\left(\epsilon|\nabla w|^{2}+\epsilon^{-1} W(w)\right) \quad \text { and } \quad E_{\epsilon}(w, D):=M_{\epsilon}(u, D)+\int_{D}|\nabla \zeta|^{2}
$$

with $\zeta$ as above, we recognize that

$$
F_{\varepsilon, \sigma}(u, \Omega)=\frac{\epsilon}{\left|\Omega_{\varrho^{-1}}\right|} E_{\epsilon}\left(w, \Omega_{\varrho^{-1}}\right)
$$

Note that $M_{\epsilon}$ is the standard scalar Ginzburg-Landau functional. The scaling (2.11) highlights the connection between the Ohta-Kawasaki functional and its sharp interface limit and gives some intuitions about the scaling factors $\epsilon$ for the energy and $\varrho$ for the periodicity.

Up to a constant factor (meaningless for what concerns the minimum problem), $E_{\epsilon}\left(\cdot, \mathbb{T}_{\varrho^{-1}}\right)$ and $F_{\varepsilon, \sigma}\left(\cdot, \mathbb{T}_{1}\right)$ are equivalent for

$$
\varrho=(\varepsilon / \sigma)^{1 / 3} \text { and } \epsilon=\varepsilon^{2 / 3} \sigma^{1 / 3}
$$

Therefore, we can consider the rescaled functional $E_{\epsilon}$ and minimize it on arbitrary tori $\mathbb{T}_{L}$, i.e. among all functions $w \in \mathscr{A}_{L}$ where

$$
\mathscr{A}_{L}:=\left\{w \in W^{1,2}\left(\mathbb{T}_{L} ;[-1,1]\right): f_{\mathbb{T}_{L}} w=m\right\} .
$$

Theorem 1 and Proposition 2.1 are, indeed, implied by the following theorem, taking $L=\varrho^{-1}$, $\gamma_{\varepsilon, \sigma}=\gamma_{\epsilon}$, with $\varrho$ and $\epsilon$ as in 2.12 (details are left to the reader).

THEOREM 2 There exist positive constants $L_{0}, \epsilon_{0}, \gamma_{\epsilon}$ and $C$ such that, for every $L_{0} \leqslant L$ and every $0<\epsilon \leqslant \epsilon_{0}$, the following holds: if $w_{\epsilon}$ is a minimizer of $E_{\epsilon}$ in $\mathbb{T}_{L}$ and $L_{0} \leqslant l \leqslant L$, then, for every $x \in \mathbb{T}_{L}$,

$$
\left|\frac{E_{\epsilon}\left(w_{\epsilon}, Q_{l}(x)\right)}{l^{n}}-\gamma_{\epsilon}\right| \leqslant C\left(\frac{1}{l}+\epsilon\right),
$$

and

$$
\left|f_{Q_{l}(x)} w_{\epsilon}-m\right| \leqslant \frac{C}{l}
$$




\subsection{The independent vector field $b$}

An equivalent formulation of Theorem 2, which will turn out to be very useful, is given by the following simple observation. If $\zeta$ is the solution of

$$
\Delta \zeta=w-m \quad \text { in } \mathbb{T}_{L}
$$

then

$$
\int_{\mathbb{T}_{L}}|\nabla \zeta|^{2}=\min \left\{\int_{\mathbb{T}_{L}}|b|^{2}: b \in L^{2}\left(\mathbb{T}_{L} ; \mathbb{R}^{n}\right), \operatorname{div} b=w-m \text { in } \mathbb{T}_{L}\right\},
$$

where $\operatorname{div} b$ is taken in the distributional sense. Moreover, the minimum is attained only in the case $b=\nabla \zeta$ (we leave the proof of these simple claims to the reader). This allows us to consider the functionals

$$
G_{\epsilon}\left(w, b, \mathbb{T}_{L}\right):=\int_{\mathbb{T}_{L}}\left(\epsilon|\nabla w|^{2}+\epsilon^{-1} W(w)+|b|^{2}\right),
$$

defined in

$$
\mathscr{B}_{L}:=\left\{(w, b): w \in W^{1,2}\left(\mathbb{T}_{L} ;[-1,1]\right), b \in L^{2}\left(\mathbb{T}_{L} ; \mathbb{R}^{n}\right), \operatorname{div} b=w-m \text { in } \mathbb{T}_{L}\right\} .
$$

We notice that the equation which defines the admissible pairs $(w, b)$ in 2.19 imposes directly the constraint on the average of $w, f_{\mathbb{T}_{L}} w=m$. Hence, using 2.17, we deduce that

$$
\min _{w \in \mathscr{A}_{L}} E_{\epsilon}\left(w, \mathbb{T}_{L}\right)=\min _{(w, b) \in \mathscr{B}_{L}} G_{\epsilon}\left(w, b, \mathbb{T}_{L}\right),
$$

with the minimum reached by the same functions $w$ and the vector field $b$ given by $b=\nabla \zeta, \zeta$ as in 2.16). Therefore, in terms of this new functional $G_{\epsilon}$, Theorem 2 reads as follows.

THEOREM 3 There exist positive constants $L_{0}, \epsilon_{0}, \gamma_{\epsilon}$ and $C$ such that, for every $L_{0} \leqslant L$ and every $0<\epsilon \leqslant \epsilon_{0}$, the following holds: if $\left(w_{\epsilon}, b_{\epsilon}\right)$ is a minimizer of $G_{\epsilon}$ in $\mathbb{T}_{L}$ and $L_{0} \leqslant l \leqslant L$, then, for every $x \in \mathbb{T}_{L}$,

and

$$
\left|G_{\epsilon}\left(w_{\epsilon}, b_{\epsilon}, Q_{l}(x)\right) / l^{n}-\gamma_{\epsilon}\right| \leqslant C\left(\frac{1}{l}+\epsilon\right)
$$

$$
\left|f_{Q_{l}(x)} w_{\epsilon}-m\right| \leqslant \frac{C}{l}
$$

The advantages of looking at minimizing pairs $\left(w_{\epsilon}, b_{\epsilon}\right)$ of $G_{\epsilon}$, instead of minimizing functions $w_{\epsilon}$ for $E_{\epsilon}$, is that $G_{\epsilon}$ is now local, the nonlocal relation having been transferred to the definition of the admissible pairs $\mathscr{B}_{L}$. This allows us to construct simple competitors for $G_{\epsilon}$, defining $w$ and $b$ locally, while for $E_{\epsilon}$ that would be impossible, since the nonlocal term $\zeta$ depends globally on $w$. This procedure is described in the following remark, which will be often used in what follows.

REMARK 2.2 Let $\Omega_{1}, \Omega_{2}$ be Lipschitz domains and $\Omega=\Omega_{1} \cup \Omega_{2}$. Take $w \in W^{1,2}(\Omega)$ and $b_{i} \in W^{1,2}\left(\Omega_{i} ; \mathbb{R}^{n}\right)$ be admissible pairs in $\Omega_{1}$ and $\Omega_{2}$, that is, $\operatorname{div} b_{i}=w-m$ in $\Omega_{i}$. Then $(w, b)$ solves $\operatorname{div} b=u-m$ in $\Omega$ if and only if the normal traces of $b_{i}$ coincide on $\partial \Omega_{1} \cap \partial \Omega_{2}$, i.e.

$$
b_{1} \cdot v=b_{2} \cdot v \quad \text { in } \partial \Omega_{1} \cap \partial \Omega_{2},
$$

where $v$ is the outer normal to $\partial \Omega_{1}$ ( $v$ will always stand for the outer normal to a domain). The proof of this assertion is immediate and is left to the reader. We point out that we will use this remark always in the following two cases: either $\Omega_{1}$ and $\Omega_{2}$ are two cubes with a common face, or $\Omega_{1}=Q_{l_{1}}$ and $\Omega_{2}=Q_{l_{2}} \backslash \bar{Q}_{l_{1}}$, with $l_{1}<l_{2}$ (see Figure 1 ). 

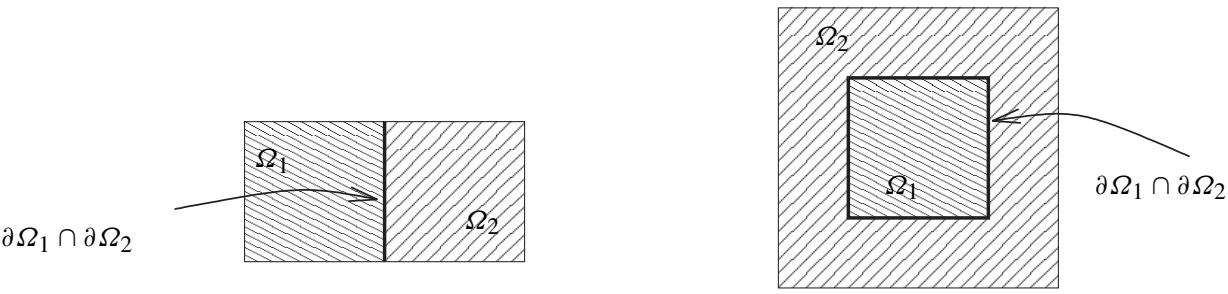

FIG. 1. For $(w, b)$ being admissible, the normal traces of $b_{i}$ have to coincide on $\partial \Omega_{1} \cap \partial \Omega_{2}$.

\subsection{Outline of the proof}

The scheme of the proof of Theorem 3 follows closely the approach of Alberti, Choksi and Otto [1]. However, in order to make the article more readable and to highlight the differences with [1], we give a sketch of the proof.

Sketch of the proof of Theorem 3 The proof is presented in Sections 3 and 4 In the first of these two sections, we develop the main ingredients for the proof, namely two comparison arguments given in Subsection 3.3, in Propositions 3.6 and 3.7 In the first of the two propositions, we compare the energy on a subdomain of a minimal pair $(w, b)$ with the minimum of the energy in the same domain, showing that they are nearly equal; then we study the dependence of this minimum energy on the size of the domain via a comparison between the minimum of $G_{\epsilon}$ in the torus and the minimum of $G_{\epsilon}$ with free boundary conditions. In this way we show that the minimum energy is almost constant for large domains, which, together with the first comparison, tells us that, given a minimal pair $(w, b)$, its energy on a subdomain is almost constant as well, thus proving the theorem.

The main ingredient in the comparisons is the construction of an interpolation between two different boundary data. Subsection 3.2 is devoted to the description of this interpolation, which is explicitly given in Corollary 3.5

The construction we describe needs an $L^{\infty}$-estimate of the independent vector field $b$ provided in Subsection 3.1. This estimate follows from a first bound on the energy in subdomains stated in Lemma 3.1 indeed, once one has an $L^{2}$-estimate of $b$ of the kind $\int_{B_{1}(x)}|b|^{2} \leqslant C$ for every point $x$, the admissibility condition $\operatorname{div} b=w-m$ gives the uniform bound via standard elliptic regularity theory. In passing, this estimate leads immediately to the proof of the uniform density distribution.

Therefore, Section 3 gives the proof of Theorem 3 under the assumption of Lemma 3.1. The proof of this lemma is given in Section 4. This is the point where our approach differs more from the one of Alberti, Choksi and Otto. Indeed, the proof of this lemma (and of Lemma 3.3, which is a slight improvement) is given by an estimate of the nonlocal part of the energy in terms of the boundary data. The bound we provide is not the optimal bound proved in [1] but an interpolation between this optimal bound and a standard $L^{2}$-bound. Even if not optimal, our estimate allows us to write a differential inequality which in turn leads to Lemma 3.1 (and Lemma 3.3). The advantage of this weaker estimate is in our opinion the proof: despite the technicalities coming from the presence of the parameter $\epsilon$, its proof is elementary, with no need of the relaxed problem and its dual formulation studied in [1].

Finally, Section 5 is devoted to proving the scaling law for the minimum energy.

REMARK 2.3 Before passing to the proof of the theorem, a comment on the notation used for the constants is in order. Throughout this paper, any constant $C$ will depend only on the volume 
fraction $m$, the dimension $n$ of the space and on the shape of the potential $W$. However, we use different notations in different contexts:

(a) the constants appearing in the statements of lemmas and propositions are indexed by the number of the statement (e.g., $C_{3.1}$ for Lemma 3.1 ): we think this will help the reader find the statement being invoked;

(b) however, this notation is only used at the first appearrance of a lemma or proposition in a proof; later in each proof we denote all the constants simply by $C$;

(c) finally, we have three special constants $C_{0}$ in (3.16), $C_{1}$ in (4.4) and $C_{2}$ in (4.18), which we need to keep track of.

\section{Uniform density and energy distribution}

In this section, we prove Theorem 3, which, in turn, gives the proof of Theorem 1 and Proposition 2.1. For clarity, we isolate the different lemmas and propositions we need for the proof in the following subsections.

\section{1 $L^{\infty}$-estimate of $b$ : uniform density distribution}

Here we prove a uniform bound for $b$ not depending on the domain $Q_{L}$ and, as a straightforward consequence, the uniform density distribution for minimizers. Using the $L^{p}$-estimates for the Laplace equation (see, for example, Theorem 9.15 in [18]: the obvious modification in the case of a torus is left to the reader), we see that a vector field $b=\nabla \zeta$ satisfying

$$
\operatorname{div} b=\Delta \zeta=w-m \in[-1-m, 1-m] \quad \text { in } \mathbb{T}_{L}
$$

belongs to $W^{1, p}\left(\mathbb{T}_{L} ; \mathbb{R}^{n}\right)$ for every $1 \leqslant p<\infty$. Hence, recalling the Sobolev embeddings, $b$ belongs to $L^{\infty}\left(\mathbb{T}_{L}\right)$, with a bound depending on the size of the domain, that is, on $L$. The key result is that, for minimizing pairs $(w, b)$ of $G_{\epsilon}$ in $\mathbb{T}_{L}$, this $L^{\infty}$-bound is independent of $L$. This estimate captures the cancellation phenomena at the base of the uniform density distribution.

We need the following lemma, which gives a preliminary estimate of the energy of minimizers on subdomains.

LEMMA 3.1 (First bound of the energy) There exist positive constants $L_{0}>2$ and $C_{3.1}$ such that, for $L \geqslant L_{0}$ and $0<\epsilon \leqslant 1 /(4 n)$, each minimizer $(w, b)$ of $G_{\epsilon}$ in $\mathbb{T}_{L}$ satisfies the following estimate: for every $L_{0} \leqslant l \leqslant L$,

$$
G_{\epsilon}\left(w, b, Q_{l}\right) / l^{n+1} \leqslant C_{\underline{3.1}} .
$$

Lemma 3.1 is based on an estimate of the nonlocal part of the energy provided in Lemma 4.1 . We postpone its proof to the next section. Here we notice that 3.2 is clearly not optimal: Theorem 3 will imply, indeed, that the energy in subdomains scales as the volume of the domain, that is, $l^{n}$. The reason why we give first this weaker estimate is merely technical: as will be clear from the proof, this allows us to take $\epsilon$ smaller than a fixed constant, $\epsilon \leqslant 1 /(4 n)$, instead of a constant depending on the domain $Q_{L}$. A kind of bootstrapping argument, passing through the uniform density distribution, allows us to recover the optimal estimate stated in Lemma 3.3 below. 
Proposition 3.2 Let $L_{0}$ be the constant in Lemma 3.1 Then there exists a constant $C_{3.2}>0$ with the following property. For every $L \geqslant L_{0}$ and $0<\epsilon \leqslant 1 /(4 n)$, each minimizer $(w, b)$ of $G_{\epsilon}$ in $\mathbb{T}_{L}$ satisfies the following uniform estimate:

$$
\|b\|_{L^{\infty}\left(\mathbb{T}_{L}\right)} \leqslant C_{3.2 .} .
$$

Proof. As already noticed, since $b=\nabla \zeta$ with $\zeta$ solving [3.1), we have $\zeta \in W^{2, p}\left(\mathbb{T}_{L}\right)$ for all $1 \leqslant p<\infty$, and therefore $b=\nabla \zeta \in L^{\infty}\left(\mathbb{T}_{L} ; \mathbb{R}^{n}\right)$. Moreover, Lemma 3.1 implies that, for every $x \in \mathbb{T}_{L}$,

$$
\int_{B_{1}(x)}|b|^{2} \leqslant C_{3.1} L_{0}^{n+1}
$$

We claim that (3.4) and the equation (3.1) give the uniform bound 3.3.

We fix $p>n$ and we start by proving that, for every $x \in \mathbb{T}_{L}$,

$$
\sup _{y \in B_{1 / 2}(x)}|b(y)| \leqslant C\left[\left(\int_{B_{1}(x)}|b|^{p}\right)^{1 / p}+1\right] .
$$

Let $\eta$ be a cut-off function such that $\eta \equiv 1$ in $B_{1 / 2}(x)$ and $\eta \equiv 0$ in $B_{1}(x)^{c}$, and set

$$
z:=(\zeta-\bar{\zeta}) \eta \quad \text { with } \bar{\zeta}=f_{B_{1}(x)} \zeta
$$

Then

$$
\Delta z=(\zeta-\bar{\zeta}) \Delta \eta+2 \nabla \zeta \cdot \nabla \eta+\Delta \zeta \eta
$$

Noticing that $\eta$ is fixed, we have

$$
\begin{aligned}
\int_{B_{1}(x)}|\Delta z|^{p} & \leqslant C\left(\int_{B_{1}(x)}|\zeta-\bar{\zeta}|^{p}+\int_{B_{1}(x)}|\nabla \zeta|^{p}+\int_{B_{1}(x)}|\Delta \zeta|^{p}\right) \\
& \stackrel{3.1}{\leqslant} C\left(\int_{B_{1}(x)}|\zeta-\bar{\zeta}|^{p}+\int_{B_{1}(x)}|\nabla \zeta|^{p}+1\right) \\
& \stackrel{\text { Poincaré }}{\leqslant} C\left(\int_{B_{1}(x)}|\nabla \zeta|^{p}+1\right) .
\end{aligned}
$$

Now, 3.6 implies that $\Delta z \in L^{p}\left(\mathbb{T}_{L}\right)$, and moreover $z=0$ on $\partial B_{1}(x)$. Hence, we can use the $L^{p}$-regularity for the Laplace equation, the Sobolev embedding and (3.7) to conclude that

$$
\|\nabla z\|_{L^{\infty}\left(B_{1}(x)\right)} \leqslant C\left\|D^{2} z\right\|_{L^{p}\left(B_{1}(x)\right)} \leqslant C\|\Delta z\|_{L^{p}\left(B_{1}(x)\right)} \leqslant C\left(\int_{B_{1}(x)}|\nabla \zeta|^{p}+1\right)^{1 / p} .
$$

From (3.8) one can deduce (3.5) noticing that $\nabla \zeta=\nabla z$ in $B_{1 / 2}(x)$.

Finally, with the use of $[3.5$ and the Young inequality, we conclude that

$$
\begin{aligned}
\|b\|_{L^{\infty}\left(\mathbb{T}_{L}\right)} & =\sup _{x \in \mathbb{T}_{L}}|\nabla \zeta(x)| \stackrel{3.5}{\leqslant} C\left[\sup _{x \in \mathbb{T}_{L}}\left(\int_{B_{1}(x)}|\nabla \zeta|^{p}\right)^{1 / p}+1\right] \\
& \leqslant C\left[\sup _{x \in \mathbb{T}_{L}}\left(\int_{B_{1}(x)}|\nabla \zeta|^{2}\right)^{1 / p} \cdot \sup _{x \in \mathbb{T}_{L}}|\nabla \zeta|^{(p-2) / p}+1\right] \\
& \stackrel{\text { Young }}{\leqslant} \frac{p-2}{p}\|b\|_{L^{\infty}\left(\mathbb{T}_{L}\right)}+C \frac{2}{p} \sup _{x \in \mathbb{T}_{L}}\left(\int_{B_{1}(x)}|\nabla \zeta|^{2}\right)^{1 / 2}+C,
\end{aligned}
$$


where we used the a priori information $b=\nabla \zeta \in L^{\infty}\left(\mathbb{T}_{L} ; \mathbb{R}^{n}\right)$. In turn, (3.8) and (3.4) imply (3.3) for a suitable constant $C_{3.2}$ depending on $C_{3.1}, L_{0}$ and $n$ (since $p$ is a fixed number greater than $n$ ).

An immediate consequence of this $L^{\infty}$-estimate is the proof of the uniform density distribution 2.21) in Theorem 3 .

Proof of 2.21 of Theorem 3 The proof reduces to the following computation: given a minimizer $\left(w_{\epsilon}, b_{\epsilon}\right)$ of $G_{\epsilon}$ in $\mathbb{T}_{L}, \epsilon \leqslant 1 /(4 n)$ and $L \geqslant L_{0}$, using Proposition 3.2, we infer

$$
\left|f_{Q_{l}(x)}\left(w_{\epsilon}-m\right)\right|=\left|f_{Q_{l}(x)} \operatorname{div} b_{\epsilon}\right| \leqslant \frac{1}{l^{n}} \int_{\partial Q_{l}(x)}\left|b_{\epsilon} \cdot v\right| \stackrel{3.3]}{\leqslant} \frac{2 n C \overline{3.2}^{n-1}}{l^{n}}=\frac{C}{l} .
$$

As pointed out before, the uniform density distribution leads to the right estimate for the energy in subdomains. We state this estimate in the following lemma and, as for the previous one, we postpone the proof to Section 4, the two estimates, indeed, are proved by the same arguments, with minor technical changes.

LEMMA 3.3 (Second bound of the energy) There exist positive constants $L_{0}>2$ and $C_{3.3}$ such that, for $L \geqslant L_{0}$ and $0<\epsilon \leqslant 1 /(4 n)$, each minimizer $(w, b)$ of $G_{\epsilon}$ in $\mathbb{T}_{L}$ satisfies the following estimate: for every $L_{0} \leqslant l \leqslant L$,

$$
G_{\epsilon}\left(w, b, Q_{l}\right) / l^{n} \leqslant G_{3.3} \text {. }
$$

\subsection{Construction procedures}

In this subsection, we describe how to construct admissible pairs with given boundary data and controlled energy. These construction procedures will be used several times to exhibit competitors in the proof of the uniform energy distribution.

The main result is the following.

LEMMA 3.4 (Basic construction) Let $d \geqslant 1, u \in W^{1,2}\left(Q_{d} ;[-1,1]\right), s \in L^{2}\left(Q_{d}\right)$ and $g \in$ $L^{2}\left(\partial Q_{d}\right)$ with

$$
\frac{1}{d^{n}}\left|\int_{Q_{d}} s+\int_{\partial Q_{d}} g\right| \leqslant 1-\alpha \in(0,1) .
$$

Then, for every $0<\epsilon \leqslant \alpha d /(4 n)$ there exist $w \in W^{1,2}\left(Q_{d} ;[-1,1]\right)$ and $b \in L^{2}\left(Q_{d} ; \mathbb{R}^{n}\right)$ such that

(i) $\left.w\right|_{\partial Q_{d}}=\left.u\right|_{\partial Q_{d}}$,

(ii) $b \cdot v=g$ on $\partial Q_{d}$,

(iii) $\operatorname{div} b=w-s$ in $Q_{d}$,

(iv) $G_{\epsilon}\left(w, b, Q_{d}\right) \leqslant M_{\epsilon}\left(u, Q_{d}\right)+C_{3.4}\left(d^{n-1}+d^{2} \int_{Q_{d}}(w-s)^{2}+d \int_{\partial Q_{d}} g^{2}\right)$,

where $C_{\overline{3.4}}$ is a constant depending only on $n$.

Proof. We start by noticing that, in order to find a vector field satisfying (ii) and (iii), it is necessary to modify $u$ to a function $w$ such that

$$
\int_{Q_{d}}(w-s)=\int_{Q_{d}} \operatorname{div} b=\int_{\partial Q_{d}} g .
$$




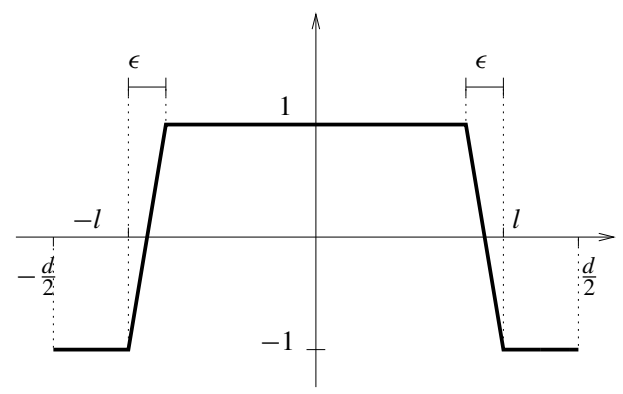

FIG. 2. The function $\phi_{l, \epsilon}$ in 1-d.

This has to be done with the trace condition (i) satisfied and controlling the Cahn-Hilliard energy of $w, M_{\epsilon}\left(w, Q_{d}\right)$. To this end, we consider the functions $\phi_{l, \epsilon}=\min \left\{\psi_{l, \epsilon}, 1\right\}$ (see Figure 2), where $l \in[0, d-\epsilon],\|x\|=\max _{i}\left|x_{i}\right|$ is the uniform norm in $\mathbb{R}^{n}$ and $\psi_{l, \epsilon}$ is given by

$$
\psi_{l, \epsilon}(x):= \begin{cases}-1 & \text { for } x \in Q_{d} \backslash Q_{2 l}, \\ 2(l-\|x\|) / \epsilon-1 & \text { for } x \in Q_{2 l} .\end{cases}
$$

We define the function $w$ by

$$
w(x)= \begin{cases}\max \left\{u(x), \phi_{l, \varepsilon}(x)\right\} & \text { if } \int_{Q_{d}} u \leqslant \int_{Q_{d}} s+\int_{\partial Q_{d}} g, \\ \min \left\{u(x),-\phi_{l, \varepsilon}(x)\right\} & \text { if } \int_{Q_{d}} u \geqslant \int_{Q_{d}} s+\int_{\partial Q_{d}} g,\end{cases}
$$

where, in both cases, $l \in[0, d-\varepsilon]$ is chosen in such a way that (3.11) holds, that is,

$$
\int_{Q_{d}} w=\int_{Q_{d}} s+\int_{\partial Q_{d}} g
$$

We notice that such a choice always exists: indeed, recalling the constraint $\epsilon \leqslant \alpha d /(4 n)$, we have

$$
\int_{Q_{d}} \phi_{d-\epsilon, \epsilon}>2(d-2 \epsilon)^{n}-d^{n} \geqslant(1-\alpha) d^{n} \stackrel{3.10}{\geqslant}\left|\int_{Q_{d}} s+\int_{\partial Q_{d}} g\right| \text {. }
$$

Moreover, by construction, $\left.w\right|_{\partial Q_{d}}=\left.u\right|_{\partial Q_{d}}$ and it is straightforward to verify that

$$
M_{\epsilon}\left(w, Q_{d}\right) \leqslant M_{\epsilon}\left(u, Q_{d}\right)+M_{\epsilon}\left(\phi_{l, \epsilon}, Q_{d}\right) \leqslant M_{\epsilon}\left(u, Q_{d}\right)+C d^{n-1} .
$$

Now, we define simply $b=\nabla v$, with $v$ solving

$$
\begin{cases}\Delta v=w-s & \text { in } Q_{d} \\ \partial v / \partial v=g & \text { on } \partial Q_{d}\end{cases}
$$

Note that by 3.13 such a $v$ does exist. Moreover, we can use the following $L^{2}$-estimate for the Laplace equation with Neumann boundary conditions:

$$
\int_{Q_{d}}|b|^{2}=\int_{Q_{d}}|\nabla v|^{2} \leqslant C\left(d^{2}\|w-s\|_{L^{2}\left(Q_{d}\right)}^{2}+d\|g\|_{L^{2}\left(\partial Q_{d}\right)}^{2}\right) .
$$

This estimate (for which we have not found a precise reference) follows from an integration by parts, and the Poincaré and trace inequalities: taking $d=1$ and $v$ with $\int_{Q_{1}} v=0$, we have 


$$
\begin{aligned}
& \int_{Q_{1}}|\nabla v|^{2} \quad=\quad-\int_{Q_{1}} \Delta v v+\int_{\partial Q_{1}} \frac{\partial v}{\partial v} v \\
& \stackrel{\text { Hölder ineq. }}{\leqslant} \quad C\left(\|w-s\|_{L^{2}\left(Q_{1}\right)}\|v\|_{L^{2}\left(Q_{1}\right)}+\|g\|_{L^{2}\left(\partial Q_{1}\right)}\|v\|_{L^{2}\left(\partial Q_{1}\right)}\right) \\
& \stackrel{\text { Poincaré and trace ineq. }}{\leqslant} C\left(\|w-s\|_{L^{2}\left(Q_{1}\right)}\|\nabla v\|_{L^{2}\left(Q_{1}\right)}+\|g\|_{L^{2}\left(\partial Q_{1}\right)}\|\nabla v\|_{L^{2}\left(\partial Q_{1}\right)}\right) \text {, }
\end{aligned}
$$

which gives 3.15 for $d=1$. The dependence of the constants on $d$ comes from a simple scaling argument.

In this way we have concluded the proof: indeed, $(w, b)$ satisfies (i)-(iii) and, recalling 3.14 and 3.15, (iv).

A corollary of Lemma 3.4 is the following interpolation construction which allows us to construct an admissible pair $(w, b)$ in a frame $Q_{l+2 d} \backslash Q_{l}$, interpolating between two boundary data. Before stating and proving it, we give the following definition.

Definition 3.1 A function $u$ belongs to $W^{1,2}\left(\partial Q_{l}\right)$ if

(a) $u \in W^{1,2}\left(T_{ \pm l, i}\right)$ for every face $T_{ \pm l, i}$

$$
T_{ \pm l, i}:=\left\{x \in Q_{l} \mid x_{i}= \pm l / 2\right\}
$$

(b) the traces of $\left.u\right|_{T_{ \pm l, j}}$ and $\left.u\right|_{T_{ \pm l, i}}$ coincide on the common edge (if it exists), i.e. on $T_{ \pm l, j} \cap T_{ \pm l, i}$.

REMARK 3.1 This is a special case of the definition of Sobolev functions on a Lipschitz manifold (see, for example, Reshetnyak [38]). We need only notice that if $u \in W^{1,2}\left(Q_{L}\right)$, then $\left.u\right|_{\partial Q_{l}} \in$ $W^{1,2}\left(\partial Q_{l}\right)$ for almost every $0<l \leqslant L$.

COROLlary 3.5 (Frame interpolation) Let $d_{0}>1$ and $C_{0}$ be constants such that

$$
\left|m+\frac{2 n C_{0}}{d_{0}}\right| \leqslant 1-\alpha \in(0,1) .
$$

Consider the following pairs of boundary data, $\left(u_{1}, g_{1}\right)$ and $\left(u_{2}, g_{2}\right)$ :

(a) $u_{1} \in W^{1,2}\left(\partial Q_{l}\right), g_{1} \in L^{\infty}\left(\partial Q_{l}\right)$, with $\left\|g_{1}\right\|_{L^{\infty}} \leqslant C_{0}$ and $l>0$;

(b) $u_{2} \in W^{1,2}\left(\partial Q_{l+2 d}\right), g_{2} \in L^{\infty}\left(\partial Q_{l+2 d}\right)$, with $\left\|g_{2}\right\|_{L^{\infty}} \leqslant C_{0}$ and $d$ given by

$$
d=l / k, \quad k \in \mathbb{N}, \quad \text { such that } d_{0} \leqslant d \leqslant 2 d_{0} .
$$

Then, for every $0<\epsilon \leqslant \alpha d_{0} /(4 n)$, there exists a pair $(w, b)$ such that

(i) $w \in W^{1,2}\left(Q_{l+2 d} \backslash Q_{l}\right), b \in L^{2}\left(Q_{l+2 d} \backslash Q_{l} ; \mathbb{R}^{n}\right)$ and

$$
\operatorname{div} b=u-m \quad \text { in } Q_{l+2 d} \backslash Q_{l} \text {; }
$$

(ii) $\left.w\right|_{\partial Q_{l}}=u_{1}$ and $\left.w\right|_{\partial Q_{l+2 d}}=u_{2}$;

(iii) $b \cdot v=g_{1}$ on $\partial Q_{l}$ and $b \cdot v=g_{2}$ on $\partial Q_{l+2 d}$;

(iv) $G_{\epsilon}\left(w, b, Q_{l+2 d} \backslash Q_{l}\right) \leqslant C\left[\frac{3.5}{\left(l^{n-1}\right.}+\epsilon^{2} \int_{\partial Q_{l}}\left|\nabla u_{1}\right|^{2}+\epsilon^{2} \int_{\partial Q_{l+2 d}}\left|\nabla u_{2}\right|^{2}\right)$,

where $C_{3.5}$ depends on $C_{0}, d_{0}$ and $n$. 


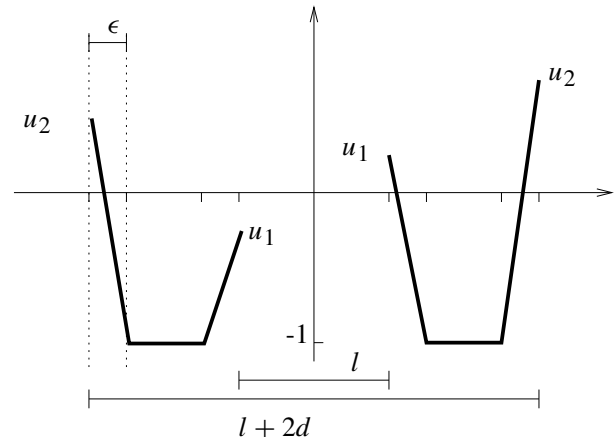

FIG. 3. The function $v$ in 1-d.

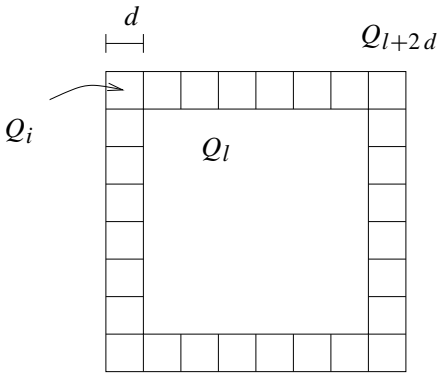

FIG. 4. Decomposition of $Q_{l+2 d} \backslash Q_{l}$ into subcubes $Q_{i}$.

Proof. Consider the function $v \in W^{1,2}\left(Q_{l+2 d} \backslash Q_{l}\right)$ interpolating between $u_{1}$ and $u_{2}$ given by (see Figure 3)

$$
v:= \begin{cases}\frac{l-\|x\|}{\epsilon}+\frac{\epsilon+l-\|x\|}{\epsilon} u_{1}\left(\frac{l x}{\|x\|}\right) & \text { in } Q_{l+\epsilon} \backslash Q_{l}, \\ -1 & \text { in } Q_{l+2 d-\epsilon} \backslash Q_{l+\epsilon}, \\ \frac{\|x\|-l-2 d}{\epsilon}+\frac{\|x\|-l-2 d+\epsilon}{\epsilon} u_{2}\left(\frac{(l+2 d) x}{\|x\|}\right) & \text { in } Q_{l+2 d} \backslash Q_{l+2 d-\epsilon} .\end{cases}
$$

It is easy to see that

$$
\left.v\right|_{\partial Q_{l}}=u_{1},\left.\quad v\right|_{\partial Q_{l+2 d}}=u_{2} \quad \text { and } \quad v \equiv-1 \quad \text { in } Q_{l+2 d-\epsilon} \backslash Q_{l+\epsilon} ;
$$

moreover, a simple computation shows that its Cahn-Hilliard energy can be estimated in the following way:

$$
\begin{aligned}
M_{\epsilon}\left(v, Q_{l+2 d} \backslash Q_{l}\right) & =\int_{Q_{l+2 d} \backslash Q_{l}}\left\{\epsilon|\nabla v|^{2}+\frac{W(v)}{\epsilon}\right\} \\
& \leqslant C\left(l^{n-1}+\epsilon^{2} \int_{\partial Q_{l}}\left|\nabla u_{1}\right|^{2}+\epsilon^{2} \int_{\partial Q_{l+2 d}}\left|\nabla u_{2}\right|^{2}\right) .
\end{aligned}
$$

Now, we divide $Q_{l+2 d} \backslash Q_{l}$ into $(k+2)^{n}-k^{n}$ cubes with side $d, Q_{i}$ (see Figure 4). For each such cube $Q_{i}$, we set

$$
\left.u_{i} \equiv v\right|_{Q_{i}} \quad \text { and } \quad g_{i}= \begin{cases}g_{1} & \text { in } \partial Q_{i} \cap \partial Q_{l}, \\ g_{2} & \text { in } \partial Q_{i} \cap \partial Q_{l+2 d} \\ 0 & \text { elsewhere }\end{cases}
$$

From 3.16, for each cube $Q_{i}$, we have

$$
\left|m+\frac{1}{d^{n}} \int_{\partial Q_{i}} g_{i}\right| \leqslant 1-\alpha \in(0,1) .
$$

So, we find pairs $\left(w_{i}, b_{i}\right)$ satisfying conclusions (i)-(iv) of Lemma 3.4 with $s \equiv m$. Therefore, we can consider the pair $(w, b)$ obtained patching together these maps, i.e. the function $w$ which 
coincides with $w_{i}$ in $Q_{i}$ and the field $b$ equal to $b_{i}$ in $Q_{i}$. It is easy to verify that the pair $(w, b)$ satisfies (i)-(iii) (we use Remark 2.2 to (i)). Moreover, from Lemma 3.4 and from the estimate (3.20, we infer (iv):

$$
\begin{gathered}
G_{\epsilon}\left(w, b, Q_{l+2 d} \backslash Q_{l}\right) \leqslant \sum_{i}^{(k+2)^{n}-k^{n}} G_{\epsilon}\left(w_{i}, b_{i}\right) \leqslant C\left[(k+2)^{n}-k^{n}\right]+M_{\epsilon}\left(v, Q_{l+2 d} \backslash Q_{l}\right) \\
\stackrel{3.20,3.17]}{\leqslant} C_{\overline{3.5}}\left(l^{n-1}+\epsilon^{2} \int_{\partial Q_{l}}\left|\nabla u_{1}\right|^{2}+\epsilon^{2} \int_{\partial Q_{l+2 d}}\left|\nabla u_{2}\right|^{2}\right),
\end{gathered}
$$

with $C_{\overline{3.5}}=C_{\overline{3.5}}\left(C_{0}, d_{0}, n\right)$.

\subsection{Proof of the main result: uniform energy distribution}

Here we prove the uniform energy distribution. The proof is based on the study of the renormalized minimum energy of $G_{\epsilon}$ in $\mathbb{T}_{l}$,

$$
\gamma_{\epsilon}(l)=\min _{(w, b) \in \mathscr{B}_{l}} G_{\epsilon}\left(w, b, \mathbb{T}_{l}\right) / l^{n}
$$

compared to the energy on subdomains.

Before proceeding with this comparison, we note that, by Lemma $3.3, \gamma_{\epsilon}(l) \leqslant C_{3.3}$ for all $\epsilon>0$ and $l>0$. Moreover, the map $l \mapsto \gamma_{\epsilon}(l)$ is continuous and, for every $\delta>0$, there exists a constant $C_{\delta}$ such that

$$
\left|\gamma_{\epsilon}(l+\delta)-\gamma_{\varepsilon}(l)\right| \leqslant C_{\delta} / l
$$

Indeed, for $(w, b) \in \mathscr{B}_{l}$, the pair $\left(w_{\lambda}, b_{\lambda}\right)$ with $w^{\lambda}(x)=w(\lambda x)$ and $b^{\lambda}(x)=\lambda^{-1} b(\lambda x)$ satisfies

$$
G_{\epsilon}\left(w^{\lambda}, b^{\lambda}, Q_{l}\right) \leqslant \max \left\{1 / \lambda^{n-2}, 1 / \lambda^{n+2}\right\} G_{\epsilon}\left(w, b, Q_{\lambda l}\right) .
$$

Hence, it is immediate to infer that $\gamma_{\epsilon}(l) \leqslant \max \left\{\lambda^{2}, 1 / \lambda^{2}\right\} \gamma_{\epsilon}(\lambda l)$, from which the claims follow.

Now, we state the first comparison argument, which asserts, roughly speaking, that the energy in subcubes $Q_{l}$ is comparable with the minimum in slightly different cubes, $\gamma_{\epsilon}(l \pm 2 d)$, where $d$ is a controlled quantity. We fix for the rest of the section a constant $d_{0}>1$ such that

$$
\left|m+\frac{2 n C[\overline{3.2}}{d_{0}}\right| \leqslant 1-\frac{1-|m|}{2}=\frac{1+|m|}{2} .
$$

Proposition 3.6 Let $L_{0}$ be as in Proposition 3.2. There exists a constant $C_{\overline{3.6}}$ such that, for all $L_{0} \leqslant l \leqslant L-4 d_{0}$, and $0<\epsilon \leqslant \epsilon_{0}=(1-|m|) d_{0} /(8 n)$, and for every minimizer $(w, b) \in \mathscr{B}_{L}$ for $G_{\epsilon}$, for some $d_{0} \leqslant d \leqslant 2 d_{0}$ we have

$$
\gamma_{\epsilon}(l+d)-C_{\overline{3.6}}(1 / l+\epsilon) \leqslant G_{\epsilon}\left(w, b, Q_{l}\right) / l^{n} \leqslant \gamma_{\epsilon}(l-d)+C_{3.6}(1 / l+\epsilon) .
$$

Proof. The two inequalities being analogous, we prove only the second. The strategy is to interpolate between a suitable trace of the minimal pair $(w, b)$ and the trace of a periodic minimal pair in a slightly smaller cube. This, indeed, allows us to construct a competitor for $(w, b)$ which gives the desired estimate. 
To choose the right trace for $(w, b)$, we recall that, from Lemma 3.3, the energy is comparable with the volume of the domain. Hence, using Fubini, we infer the existence of $l_{1} \in[l, l+1]$ such that

$$
\epsilon \int_{\partial Q_{l_{1}}}|\nabla w|^{2} \leqslant C_{[3.3]^{n}} \leqslant C l^{n} .
$$

Consider, then, a minimal pair $(s, z)$ in $\mathscr{B}_{l_{1}-2 d}$, i.e. a pair realizing $\gamma_{\epsilon}\left(l_{1}-2 d\right)$, where $d$ is determined by $l_{1}$ in the following way:

$$
d=l_{1} / k, k \in \mathbb{N}, \quad \text { such that } d_{0} \leqslant d \leqslant 2 d_{0} .
$$

Again from Lemma 3.3, we may assume, without loss of generality (possibly we have to translate our minimal pair), that

$$
\epsilon \int_{\partial Q_{l_{1}-2 d}}|\nabla s|^{2} \leqslant C_{[3.3 .3}\left(l_{1}-2 d\right)^{n-1} \leqslant C l^{n-1} .
$$

Moreover, since $(w, b)$ and $(s, z)$ are minimizers, $b$ and $z$ are both gradients of $W^{2, p}$ functions (so, in particular, they do possess a normal trace) and, by Proposition 3.2 they are uniformly bounded by $C_{3.2}$

Hence, from 3.23 , we can apply Corollary 3.5 with

$$
u_{1}=s, g_{1}=z \cdot v \quad \text { on } \partial Q_{l_{1}-2 d} \quad \text { and } \quad u_{2}=w, g_{2}=b \cdot v \quad \text { on } \partial Q_{l_{1}} \text {, }
$$

obtaining a pair $(\bar{w}, \bar{b})$ in $Q_{l_{1}} \backslash Q_{l_{1}-2 d}$ satisfying conclusions (i)-(iv) of Corollary 3.5 This implies (see Remark 2.2) that the pair which is equal to $(s, z)$ in $Q_{l_{1}-2 d}$ and to $(\bar{w}, \bar{b})$ in $Q_{l_{1}} \backslash Q_{l_{1}-2 d}$ is admissible. Therefore, we deduce the desired inequality,

$$
\begin{aligned}
& G_{\epsilon}\left(w, b, Q_{l}\right) \leqslant G_{\epsilon}\left(w, b, Q_{l_{1}}\right) \leqslant G_{\epsilon}\left(s, z, Q_{l_{1}-2 d}\right)+G_{\epsilon}\left(\bar{w}, \bar{b}, Q_{l_{1}} \backslash Q_{l_{1}-2 d}\right) \\
& \stackrel{\text { Cor. B..5 }}{\leqslant}\left(l_{1}-2 d\right)^{n} \gamma_{\epsilon}\left(l_{1}-2 d\right)+C l^{n-1}+\epsilon^{2} \int_{\partial Q_{l_{1}-2 d}}\left|\nabla s_{\epsilon}\right|^{2}+\epsilon^{2} \int_{\partial Q_{l_{1}}}|\nabla w|^{2} \\
& \left.\leqslant l^{n} \gamma_{\epsilon}(l-d)+C_{[3.6}\right]^{n-1}+C_{[3.6]} l^{n},
\end{aligned}
$$

where we use $l_{1}-2 d \leqslant l-d$ and 3.22 .

The next proposition deals with the asymptotic behavior of the quantities $\gamma_{\epsilon}(l)$. To this end, set

$$
\mathscr{C}_{l}:=\left\{(w, b) \mid w \in W^{1,2}\left(Q_{l} ;[-1,1]\right), b \in L^{2}\left(Q_{l} ; \mathbb{R}^{n}\right), \operatorname{div} b=u-m,\|b\|_{L^{\infty}} \leqslant G_{\text {[3.2 }}\right\},
$$

where the equation $\operatorname{div} b=u-m$ is understood in the distributional sense with free boundary conditions; and denote by $\alpha_{\epsilon}(l)$ the normalized minimum in this class,

$$
\alpha_{\epsilon}(l):=\min _{(u, b) \in \mathscr{C}_{l}} G_{\epsilon}\left(u, b, Q_{l}\right) / l^{n} .
$$

Then the following proposition holds. 
Proposition 3.7 Let $L_{0}$ and $\epsilon_{0}$ be as in Proposition 3.6. Then, for every $L_{0} \leqslant l$ and $0 \leqslant \epsilon \leqslant \epsilon_{0}$, the following conclusions hold:

(i) $\alpha_{\epsilon}(l) \leqslant \alpha_{\epsilon}(k l)$ and $\gamma_{\epsilon}(k l) \leqslant \gamma_{\epsilon}(l)$, for every $k \in \mathbb{N}$;

(ii)

$$
\alpha_{\epsilon}(l) \leqslant \gamma_{\epsilon}(l) \leqslant \alpha_{\epsilon}(l)+C(1 / l+\epsilon),
$$

(iii) there exist constants $\gamma_{\epsilon}>0$ such that

$$
\left|\gamma_{\epsilon}(l)-\gamma_{\epsilon}\right| \leqslant G_{\overline{3.7}}(1 / l+\epsilon) .
$$

Proof. Conclusion (i) follows from simple comparisons. Let $(w, b)$ be a minimal pair for $\alpha_{\epsilon}(k l)$; then its restrictions to subcubes of side $l$, say $Q_{i}$ for $i=1, \ldots, k^{n}$, belong to $\mathscr{C}_{l}$. Therefore,

$$
(k l)^{n} \alpha_{\epsilon}(k l)=\sum_{i=1}^{k^{n}} G_{\epsilon}\left(w, b, Q_{i}\right) \geqslant k^{n} l^{n} \alpha_{\epsilon}(l) .
$$

Similarly, let $(w, b)$ be a minimal pair for $\gamma_{\epsilon}(l)$ and extend it periodically on $Q_{k l}$. In this way we obtain a pair in $\mathscr{B}_{k l}$, which we denote $(\bar{w}, \bar{b})$, so that

$$
(k l)^{n} \gamma_{\epsilon}(k l) \leqslant G_{\epsilon}\left(\bar{w}, \bar{b}, Q_{k l}\right)=\sum_{i=1}^{k^{n}} G_{\epsilon}\left(w, b, Q_{i}\right)=(k l)^{n} \gamma_{\epsilon}(l)
$$

For (ii), taking into account Proposition 3.2 we note that the minimal pairs for $G_{\epsilon}$ in $\mathscr{B}_{l}$ belong to $\mathscr{C}_{l}$. Therefore, one infers immediately that $\alpha_{\epsilon}(l) \leqslant \gamma_{\epsilon}(l)$. The right inequality of 3.29) follows, instead, from the same arguments used in the proof of Proposition 3.6. Let $(w, b)$ be a minimal pair for $\alpha_{\epsilon}(l)$; since $\alpha_{\epsilon}(l) \leqslant C l^{n}$, there exists $l_{1} \in[l-1, l]$ such that

$$
\epsilon \int_{\partial Q_{l_{1}}}|\nabla w|^{2} \leqslant C_{[\overline{3.3}}{ }^{n}
$$

Moreover, let $d \in\left[d_{0}, 2 d_{0}\right]$ be such that $l_{1}=k d$ and $k \in \mathbb{N}$. An interpolation between the boundary values of $(w, b)$ on $\partial Q_{l_{1}}$ and periodic values on $\partial Q_{l_{1}+2 d}$ (e.g. homogeneous data) leads to (see the proof of Proposition 3.6 for details)

$$
\gamma_{\epsilon}(l) \stackrel{3.22}{\leqslant} \gamma_{\epsilon}\left(l_{1}+2 d\right)+C / l \leqslant \alpha_{\epsilon}\left(l_{1}\right)+C(1 / l+\epsilon) \leqslant \alpha_{\epsilon}(l)+C(1 / l+\epsilon) .
$$

Finally, for (iii), we fix $\gamma_{\epsilon}:=\lim \sup _{l \rightarrow+\infty} \alpha_{\epsilon}(l)$. Then, for every $l$ and $\epsilon$, we have

$$
\alpha_{\epsilon}(l) \leqslant \gamma_{\epsilon} \leqslant \gamma_{\epsilon}(l) \text {. }
$$

Indeed, from (i) we infer that

$$
\alpha_{\epsilon}(l) \leqslant \limsup _{k \rightarrow+\infty} \alpha_{\epsilon}(k l) \leqslant \limsup _{l \rightarrow+\infty} \alpha_{\epsilon}(l)=\gamma_{\epsilon} \leqslant \liminf _{l \rightarrow+\infty} \gamma_{\epsilon}(l) \leqslant \liminf _{k \rightarrow+\infty} \gamma_{\epsilon}(k l) \leqslant \gamma_{\epsilon}(l) .
$$

Hence, from 3.29) and 3.32, we deduce 3.30:

$$
0 \leqslant \gamma_{\epsilon}(l)-\gamma_{\epsilon} \leqslant \gamma_{\epsilon}(l)-\alpha_{\epsilon}(l) \leqslant C_{\overline{3.7}}(1 / l+\epsilon) .
$$


It remains to prove that $\gamma_{\epsilon}>0$. For the monotonicity of $\alpha_{\epsilon}(k l)$, it is enough to verify that $\alpha_{\epsilon}(l)>0$ for some $l$. This follows easily: indeed, otherwise

$$
\int_{Q_{l}} W(u)=0
$$

that is, $u \equiv \pm 1$, contrary to the assumption $m \in(-1,1)$.

Using Propositions 3.6 and 3.7 we can conclude the proof of Theorem 3

Proof of 2.20 of Theorem 3 We take $L_{0}$ and $\epsilon_{0}$ as in Proposition 3.6. and let $\gamma_{\epsilon}$ be the constants in Proposition 3.7. Then, for any $L_{0}+1 \leqslant l \leqslant L-4 d_{0}$ and $\epsilon \leqslant \epsilon_{0}$, applying the right hand inequality of 3.24) and (3.30, we find

$$
G_{\epsilon}\left(w_{\epsilon}, b_{\epsilon}, Q_{l}\right) / l^{n}-\gamma_{\epsilon} \stackrel{\sqrt[3.24]{\leqslant}}{\leqslant} \gamma_{\epsilon}(l-d)-\gamma_{\epsilon}+C_{3.6(1 / l+\epsilon)} \stackrel{3.30}{\leqslant} C(1 / l+\epsilon) .
$$

In the same way, using the left inequality of (3.24), we get

$$
G_{\epsilon}\left(w_{\epsilon}, b_{\epsilon}, Q_{l}\right) / l^{n}-\gamma_{\epsilon} \geqslant-C(1 / l+\epsilon) .
$$

Clearly, 3.33 and 3.34) complete the proof for $l \leqslant L-4 d_{0}$. When $l \geqslant L-4 d_{0}$, notice simply that

$$
G_{\epsilon}\left(w_{\epsilon}, b_{\epsilon}, Q_{L-4 d_{0}}\right) /\left(L-4 d_{0}\right)^{n}-C / l \leqslant G_{\epsilon}\left(w_{\epsilon}, b_{\epsilon}, Q_{l}\right) / l^{n} \leqslant \gamma_{\epsilon}(L)+C / l,
$$

and the conclusion follows.

\section{Proof of the basic estimate}

This section is devoted to the proof of Lemmas 3.1 and 3.3 The proof of the latter is much similar to that of the former: it is obtained by the same arguments with the help of Proposition 3.2 established in the previous section. Both are based on an estimate of the nonlocal part of the energy, given in Lemma 4.1. which is the point where our arguments differ most from those in [1]; this estimate, although weaker than the one in [1], is sufficient and, in our opinion, clearer and cleaner.

Lemma 4.1 (Nonlocal energy estimate) For every $\delta>0$, there exists a constant $G_{\overline{4.1}(}(\delta)$ with the following property. Let $g \in L^{2}\left(\partial Q_{L}\right), L>0$, satisfy

$$
\left|\frac{1}{L^{n}} \int_{\partial Q_{L}} g+m\right| \leqslant 1-\alpha \quad \text { with } \alpha \in(0,1) .
$$

Then there exists a vector field $b \in L^{2}\left(Q_{L} ; \mathbb{R}^{n}\right)$ such that:

(i) $b \cdot v=g$ on $\partial Q_{L}$,

(ii) $\operatorname{div} b \in L^{\infty}\left(Q_{L}\right)$ with $|\operatorname{div} b+m| \leqslant 1-\alpha$,

(iii)

$$
\int_{Q_{L}}|b|^{2} \leqslant \delta L \int_{\partial Q_{L}} g^{2}+q_{[4.1]}(\delta)\left(\int_{\partial Q_{L}} g^{2}\right)^{\frac{n+2}{n+1}} .
$$


Proof. We start with a simple observation: for every $d>0$ and $h \in L^{2}\left(\partial Q_{d}\right)$, the vector field $b=\nabla v$, with $v$ solving

$$
\begin{cases}\Delta v=\frac{1}{d^{n}} \int_{\partial Q_{d}} h & \text { in } Q_{d} \\ \frac{\partial v}{\partial v}=h & \text { on } \partial Q_{d}\end{cases}
$$

satisfies the following estimate (see, for example, [17]):

$$
\int_{Q_{d}}|b|^{2}=\int_{Q_{d}}|\nabla v|^{2} \leqslant C\left(\frac{d^{2}}{d^{n}}\left(\int_{\partial Q_{d}} h\right)^{2}+d \int_{\partial Q_{d}} h^{2}\right) \leqslant C_{1} d \int_{\partial Q_{d}} h^{2}
$$

(here we have also used Jensen's inequality).

In order to improve (4.4) to (4.2], we show that, for every $\delta>0$, there exists $\varepsilon(\delta)>0$ with the following property: if $\int_{\partial Q_{L}} g^{2} \leqslant \varepsilon(\delta) L^{n+1}$, then there exists a vector field $b \in L^{2}\left(Q_{L} ; \mathbb{R}^{n}\right)$ satisfying (i), (ii) and

$$
\int_{Q_{L}}|b|^{2} \leqslant \delta L \int_{\partial Q_{L}} g^{2}
$$

To this end, let $k \in \mathbb{N}$ be the smallest integer such that $d_{0}=k^{-1} \leqslant \delta / C_{1}$, where $C_{1}$ is the constant in 4.4, and set

$$
\varepsilon(\delta)=\frac{(1-\alpha-m)^{2} d_{0}^{2 n}}{2 n} .
$$

Consider now the frame $Q_{L} \backslash Q_{L-2 d}$ decomposed into the union of $k^{n}-(k-2)^{n}$ cubes of side $d=L d_{0}$ as in Figure 4 (with $l$ replaced by $L-2 d$ ), $Q_{L} \backslash Q_{L-2 d}=\bigcup_{i} Q_{i}$. For every cube $Q_{i}$, consider the function $g_{i} \in L^{2}\left(\partial Q_{i}\right)$ given by

$$
g_{i}= \begin{cases}g & \text { on } \partial Q_{i} \cap \partial Q_{L} \\ 0 & \text { elsewhere }\end{cases}
$$

An easy computation gives

$$
\frac{1}{d^{n}}\left|\int_{\partial Q_{i}} g_{i}\right| \leqslant \frac{1}{d^{n}} \int_{\partial Q_{L}}|g| \leqslant \frac{\sqrt{2 n L^{n-1}}}{d^{n}}\left(\int_{\partial Q_{L}} g^{2}\right)^{1 / 2} \leqslant \sqrt{\frac{2 n \varepsilon(\delta) L^{2 n}}{d^{2 n}}} \stackrel{4.6}{=} 1-\alpha-m .
$$

Hence, for every $Q_{i}$, using (4.4), there exists a vector field $b_{i} \in L^{2}\left(Q_{i} ; \mathbb{R}^{n}\right)$ such that

$$
b_{i} \cdot v=g_{i} \quad \text { on } \partial Q_{i}, \quad\left|\operatorname{div} b_{i}+m\right|=\left|m+\frac{1}{d^{n}} \int_{\partial Q_{i}} g_{i}\right| \stackrel{4.8}{\leqslant} 1-\alpha
$$

and

$$
\int_{Q_{i}}\left|b_{i}\right|^{2} \stackrel{4.4}{\leqslant} C d \int_{\partial Q_{i}} g_{i}^{2}
$$

Therefore, recalling Remark 2.2 and using (4.9), 4.10, we infer that the vector field $b$ given by $b_{i}$ in $Q_{i}$ and 0 elsewhere satisfies (i), (ii) and (4.5):

$$
\int_{Q_{L}}|b|^{2}=\sum_{i=1}^{k^{n}-(k-2)^{n}} \int_{Q_{i}}\left|b_{i}\right|^{2} \leqslant \sum_{i=1}^{k^{n}-(k-2)^{n}} C d \int_{\partial Q_{i}} g_{i}^{2} \leqslant \delta L \int_{\partial Q_{L}} g^{2} .
$$


Hence, setting $G_{4.1}(\delta):=C_{1} / \varepsilon(\delta)^{1 /(n+1)}$, we can conclude the proof. Indeed, given $g \in L^{2}\left(\partial Q_{L}\right)$ and $\delta>0$, two cases can occur:

(a) either $\int_{\partial Q_{L}} g^{2}>\varepsilon(\delta) L^{n+1}$, in which case, using [4.4), we deduce the existence of a vector field $b \in L^{2}\left(Q_{L} ; \mathbb{R}^{n}\right)$ satisfying (i), (ii) and the estimate

$$
\int_{Q_{L}}|b|^{2} \leqslant C_{1} L \int_{\partial Q_{L}} g^{2} \leqslant C_{4.1}(\delta)\left(\int_{\partial Q_{L}} g^{2}\right)^{\frac{n+2}{n+1}} ;
$$

(b) or $\int_{\partial Q_{L}} g^{2} \leqslant \varepsilon(\delta) L^{n+1}$, in which case there exists a vector field $b$ satisfying (i), (ii) and 4.5. In both cases, therefore, there exists $b$ satisfying (i)-(iii).

Next, we describe a second procedure to construct competitor pairs $(w, b)$ given boundary data $\left.w\right|_{\partial Q_{L}}=\left.u\right|_{\partial Q_{L}}$ and $b \cdot v=g$. Compared with Lemma 3.4 this new construction gives a better estimate in $L$, the side of the domain. It is obtained by applying Lemma 3.4 to a suitable subdivision of $Q_{L}$.

LEMMA 4.2 (Competitors' construction) Let $L \geqslant 2, g \in L^{2}\left(\partial Q_{L}\right)$ and $u \in W^{1,2}\left(Q_{L}\right)$, with

$$
\left|\frac{1}{L^{n}} \int_{\partial Q_{L}} g+m\right| \leqslant 1-\alpha \in(0,1)
$$

Then, for every $\delta>0$ and for every $\epsilon \leqslant \alpha \sqrt{L} /(4 n)$, there exist $w \in W^{1,2}\left(Q_{L}\right)$ and $b \in$ $L^{2}\left(Q_{L} ; \mathbb{R}^{n}\right)$ such that:

(i) $\begin{cases}\operatorname{div} b=w-m & \text { in } Q_{L} \\ b \cdot v=g & \text { on } \partial Q_{L}\end{cases}$

(ii) $\left.w\right|_{\partial Q_{L}}=\left.u\right|_{\partial Q_{L}}$;

(iii) $G_{\epsilon}\left(w, b, Q_{L}\right) \leqslant 2 \delta L \int_{\partial Q_{L}} g^{2}+2 G_{\overline{4.1}}(\delta)\left(\int_{\partial Q_{L}} g^{2}\right)^{\frac{n+2}{n+1}}+G_{4.2 .} L^{n+1}$

$$
+2 \epsilon^{2} \int_{\partial Q_{L}}|\nabla u|^{2}
$$

Moreover, if $\epsilon \leqslant \alpha /(4 n)$, (iii) can be improved to

$$
\text { (iii) } \begin{aligned}
G_{\epsilon}\left(w, b, Q_{L}\right) \leqslant & 2 \delta L \int_{\partial Q_{L}} g^{2}+2 C_{\overline{4.1}}(\delta)\left(\int_{\partial Q_{L}} g^{2}\right)^{\frac{n+2}{n+1}}+C_{\overline{4.2}} L^{n} \\
& +2 \epsilon^{2} \int_{\partial Q_{L}}|\nabla u|^{2} .
\end{aligned}
$$

Proof. Consider a vector field $\bar{b}$ as in Lemma 4.1, that is, such that

$\left(\mathrm{a}_{1}\right) \bar{b} \cdot v=g \quad$ on $\partial Q_{L}, \quad|\operatorname{div} \bar{b}+m| \leqslant 1-\alpha$,

(b $) \int_{Q_{L}}|\bar{b}|^{2} \leqslant \delta L \int_{\partial Q_{L}} g^{2}+C_{4.1}(\delta)\left(\int_{\partial Q_{L}} g^{2}\right)^{\frac{n+2}{n+1}}$. 
Consider the function $\bar{w} \in W^{1,2}\left(Q_{L}\right)$ given by

$$
\bar{w}:= \begin{cases}\frac{\|x\|-L / 2}{\epsilon}+\frac{\epsilon-L / 2+\|x\|}{\epsilon} u\left(\frac{L x}{2\|x\|}\right) & \text { in } Q_{L} \backslash Q_{L-2 \epsilon}, \\ -1 & \text { in } Q_{L-2 \epsilon},\end{cases}
$$

where, we recall, $\|x\|:=\max _{i}\left|x_{i}\right|$. It is immediate to verify that $\left.\bar{w}\right|_{\partial Q_{L}}=\left.u\right|_{\partial Q_{L}}$ and

$$
M_{\epsilon}(\bar{w}) \leqslant C L^{n-1}+\epsilon^{2} \int_{\partial Q_{L}}|\nabla u|^{2}
$$

Now, it is evident that the pair $(\bar{w}, \bar{b})$ satisfies (ii) and (iii) of the lemma; we want to modify it to a pair $(w, b)$ satisfying (i) as well. To this end, divide $Q_{L}$ into subcubes of size $\sqrt{L}$ : namely, take $k \in \mathbb{N}$ such that $k \leqslant \sqrt{L}<k+1$ and consider cubes $Q_{i}$ of side $d=L / k$, so that $\sqrt{L} \leqslant d<$ $L /(\sqrt{L}-1) \leqslant 4 \sqrt{L}$, for $L \geqslant 2$. We can, hence, apply Lemma 3.4 with $\left.\bar{w}\right|_{Q_{i}}, s=\operatorname{div} \bar{b}+m$ and $g=0$, obtaining pairs $\left(w_{i}, b_{i}\right)$ in $Q_{i}$ such that

$$
\begin{gathered}
\text { (a } \left.\mathrm{a}_{2}\right)\left.w_{i}\right|_{\partial Q_{i}}=\left.\bar{w}\right|_{\partial Q_{i}}, \quad \bar{b}_{i} \cdot v=0 \quad \text { on } \partial Q_{i}, \quad \operatorname{div} b_{i}=w_{i}-\operatorname{div} \bar{b}-m, \\
\left(\mathrm{~b}_{2}\right) G_{\epsilon}\left(w_{i}, b_{i}, Q_{i}\right) \leqslant M_{\varepsilon}\left(\bar{w}, Q_{i}\right)+C_{3.4}\left(d^{n-1}+d^{2}\left\|w_{i}-\operatorname{div} \bar{b}-m\right\|_{L^{2}\left(Q_{i}\right)}\right) \\
\leqslant M_{\varepsilon}\left(\bar{w}, Q_{i}\right)+C L^{n / 2+1} .
\end{gathered}
$$

We can now define the final pair $(w, b)$ by

$$
(w, b)=\left(w_{i}, \bar{b}+b_{i}\right) \quad \text { in } Q_{i} .
$$

Since $\left.w_{i}\right|_{\partial Q_{i}}=\left.\bar{w}\right|_{\partial Q_{i}}$, it is immediate to verify that $w$ belongs to $W^{1,2}\left(Q_{L}\right)$ and (ii) holds; moreover, applying Remark 2.2 repeatedly and $\left(\mathrm{a}_{2}\right)$, one immediately infers that (i) holds.

For (iii), instead, we use $\left(\mathrm{b}_{1}\right), 4.16$ and $\left(\mathrm{b}_{2}\right)$ to conclude that (recall $k \leqslant \sqrt{L}$ )

$$
\begin{aligned}
G_{\epsilon}\left(w, b, Q_{L}\right) & =\sum_{i=1}^{k^{n}} M_{\epsilon}\left(w_{i}, Q_{i}\right)+2 \int_{Q_{i}}\left(|\bar{b}|^{2}+\left|b_{i}\right|^{2}\right)=2 \sum_{i=1}^{k^{n}} G_{\epsilon}\left(w_{i}, b_{i}, Q_{i}\right)+2 \int_{Q_{L}}|\bar{b}|^{2} \\
& \leqslant 2 M_{\epsilon}\left(\bar{w}, Q_{L}\right)+2 \int_{Q_{L}}|\bar{b}|^{2}+C k^{n} L^{n / 2+1} \\
& \leqslant 2 \delta L \int_{\partial Q_{L}} g^{2}+2 C_{\overline{4.1}}(\delta)\left(\int_{\partial Q_{L}} g^{2}\right)^{\frac{n+2}{n+1}}+C_{\overline{4.2}} L^{n+1}+2 \epsilon^{2} \int_{\partial Q_{L}}|\nabla u|^{2} .
\end{aligned}
$$

In the case $\epsilon \leqslant \alpha /(4 n)$, we can consider a subdivision $Q_{i}$ into cubes of side 1 and apply Lemma 3.4 to this subdivision. It is not difficult to see that (iii) ${ }^{\prime}$ follows.

REMARK 4.1 We stress here the role of the bound $\epsilon \leqslant \alpha \sqrt{L} /(4 n)$ in the proof of the weaker estimate 4.13). The reason for such a bound is a technicality we will meet in the proof of Lemma 3.1. the same which does not allow us to recover immediately the optimal estimate given in Lemma 3.3

Using the previous two lemmas, we give the proof of Lemma 3.1 (restated here for the reader's convenience). 
LEMmA 3.1 There exist positive constants $L_{0}$ and $C_{\text {3.1. }}$ such that, for $L \geqslant L_{0}$ and $0<\epsilon \leqslant 1 /(4 n)$, each minimizer $(w, b)$ of $G_{\epsilon}$ in $\mathbb{T}_{L}$ satisfies the following estimate: for every $L_{0} \leqslant l \leqslant L$,

$$
G_{\epsilon}\left(w, b, Q_{l}\right) / l^{n+1} \leqslant C_{3.1} .
$$

Proof. Let $(w, b)$ be a minimal pair for $G_{\epsilon}$ in $Q_{L}$ and $f(l):=G_{\epsilon}\left(w, b, Q_{l}\right)$. First we notice that there exists a constant $C_{2}$ such that $f(L) \leqslant C_{2} L^{n} \leqslant C_{2} L^{n+1}$. Consider, indeed, the onedimensional competitor $w(x)=u\left(x_{1}\right)$, where $u$ is the piecewise linear periodic function in Figure 5 . Then

$$
E_{\epsilon}\left(w, Q_{L}\right)=L^{n-1} E_{\epsilon}(u)=L^{n-1} \int_{-L / 2}^{L / 2}\left(\epsilon\left|u^{\prime}\right|^{2}+\epsilon^{-1} W\left(u^{\prime}\right)+\left|v^{\prime}\right|^{2}\right) \leqslant C_{2} L^{n},
$$

where $v$ solves $v^{\prime \prime}=u-m$ with periodic boundary condition (see Figure 6) 1 Moreover, an easy

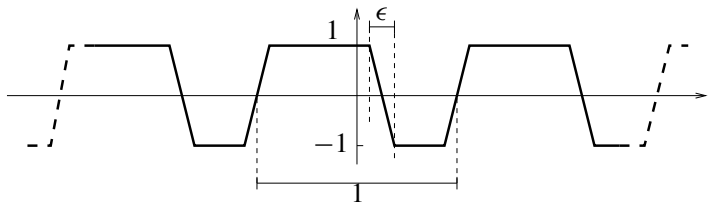

FIG. 5. The function $u$.

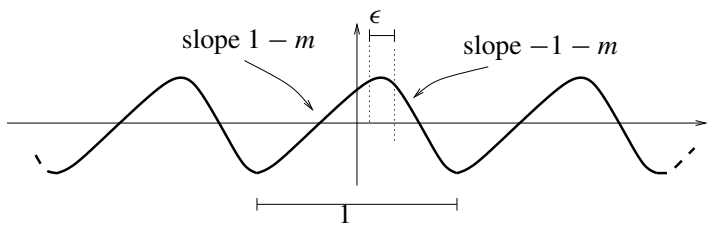

FIG. 6. The function $v$.

computation, using Fubini's theorem, yields, for $l \in(0, L)$,

$$
\begin{aligned}
f^{\prime}(l)^{+} & =\limsup _{h \rightarrow 0^{+}} \frac{f(l+h)-f(l)}{h}=\limsup _{h \rightarrow 0^{+}} \int_{Q_{l+h} \backslash Q_{l}}\left(\epsilon|\nabla u|^{2}+|b|^{2}\right) \\
& \geqslant \int_{\partial Q_{l}}\left(\epsilon|\nabla u|^{2}+(b \cdot v)^{2}\right),
\end{aligned}
$$

where we have used $b \in W^{1, p}\left(Q_{L} ; \mathbb{R}^{n}\right)$, for every $1 \leqslant p<\infty$, so it has a well defined normal trace on $\partial Q_{l}$.

Next, we observe that if

$$
\left|f_{Q_{l}} w\right|=\left|m+\frac{1}{l^{n}} \int_{\partial Q_{l}} b \cdot v\right|<1-\frac{1}{\sqrt{l}},
$$

1 Alternatively, one can consider the one-dimensional minimizer $u$, whose energy can be proved to be a multiple of the length of the domain (as shown, for example, in [6] and [31]). 
then we can apply Lemma 4.2 in $Q_{l}$, with $\alpha=1 / \sqrt{l}$ and $g=b \cdot v$. Hence, for all $\delta>0$ and $\epsilon \leqslant 1 /(4 n)=\alpha \sqrt{l} /(4 n)$, there exists a pair $(\bar{w}, \bar{b})$ satisfying (i)-(iii) of the lemma. This is where we profit from the possibility to take $\epsilon \leqslant \alpha \sqrt{l} /(4 n)$ in Lemma 4.2 Therefore, since $\left.(w, b)\right|_{Q_{l}}$ is a minimizer in $Q_{l}$ among all pairs having the same boundary data (both for $w$ and $g=b \cdot v$ ), we infer that, for every $\delta>0$,

$$
\begin{aligned}
f(l)=G_{\epsilon}\left(w, b, Q_{l}\right) & \leqslant G_{\epsilon}\left(\bar{w}, \bar{b}, Q_{l}\right) \\
& \leqslant 2 \delta l \int_{\partial Q_{l}} g^{2}+2 C_{4.1}(\delta)\left(\int_{\partial Q_{l}} g^{2}\right)^{\frac{n+2}{n+1}}+\left.C_{[4.2}\right|^{n+1}+2 \epsilon^{2} \int_{\partial Q_{l}}|\nabla u|^{2} \\
& \left.\stackrel{4.19]}{\leqslant} 2 \delta l f^{\prime}(l)^{+}+2 C_{4.1}(\delta)\left(f^{\prime}(l)^{+}\right)^{\frac{n+2}{n+1}}+C_{[4.2}\right]^{n+1}+2 \epsilon f^{\prime}(l)^{+} .
\end{aligned}
$$

On the other hand, if 4.20 is not satisfied, we claim that there exist constants $G_{6.1}$ and $R_{0}$ such that, for every $l \geqslant R_{0}$,

$$
\left.f(l) \geqslant C_{6.1}\right]^{n+2} \text {. }
$$

To see this, suppose that $w-m \geqslant c>0$ in $Q_{l}$ or $w-m \leqslant-c<0$, for some fixed $c$; then, since $\operatorname{div} b=w-m$, the maximum principle would give immediately (4.22). In the general case, we do not have $w-m \geqslant c>0$ or $w-m \leqslant-c<0$; but, since $\left|f_{Q_{l}} w\right| \geqslant 1-1 / \sqrt{l}$, this is true on a large set (in a measure-theoretic sense), so we can still argue as above via the maximum principle. We postpone the proof of this technical statement to Lemma 6.1 in the Appendix.

Now, we are ready to conclude the proof. We take $C_{\overline{3.1}}=4 \max \left\{C_{4.2}, C_{2}\right\}$ and set

$$
D_{0}:=\max \left\{C_{2} / C_{\overline{6.1},}, R_{0}\right\} \quad \text { and } \quad R=\max \left\{l \in\left[D_{0}, L\right]:\left|f_{Q_{l}} w\right| \geqslant 1-1 / \sqrt{l}\right\},
$$

where $C_{2}$ and $C_{6.1}$ are the constants in 4.18 and 4.22 . Let $\lambda \geqslant R$ be the smallest number such that

$$
\left.f(l) \leqslant C_{[3.1}\right]^{n+1} \quad \text { for every } l \in[\lambda, L] .
$$

Note that if $L_{0}>D_{0}$, then $R \leqslant \lambda<L$ : indeed, if $R=L$, we would have

$$
C_{2} L^{n+2} \stackrel{4.22}{\leqslant} f(L) \leqslant C_{1} L^{n+1}, \quad \text { i.e. } L \leqslant C_{1} / C_{2} \leqslant D_{0}<L_{0} ;
$$

and, on the other hand, since $f(L) \leqslant C_{3.1} L^{n} / 4 \leqslant C_{3.1} L^{n+1}$, by continuity, $\lambda<L$. Clearly, two cases can occur: either $R=\lambda$ or $R<\lambda$. In the former case,

$$
C_{6.1} R^{n+2} \stackrel{4.22}{\leqslant} f(R) \stackrel{4.23}{\leqslant} C_{3.1} R^{n+1},
$$

so that $R \leqslant G_{3.1} / C_{6.1}$. In the latter, as by continuity,

$$
f(\lambda)=C_{3.1} \lambda^{n+1} \text { and } f^{\prime}(\lambda)^{+} \leqslant(n+1) C_{3.1} \lambda^{n},
$$

we can use 4.21) (valid for $l>R$ ) to deduce that

$$
\begin{aligned}
C_{3.11} \lambda^{n+1}=f(\lambda) \leqslant & 2 \delta \lambda f^{\prime}(\lambda)+2 C_{4.1}(\delta) f^{\prime}(\lambda)^{\frac{n+2}{n+1}}+C_{4.2} \lambda^{n+1}+2 \epsilon f^{\prime}(\lambda) \\
\leqslant & 2 \delta(n+1) C_{3.1} \lambda^{n+1}+2 C_{4.1}(\delta)\left((n+1) C_{3.1} \lambda^{n}\right)^{\frac{n+2}{n+1}} \\
& +C_{4.2} \lambda^{n+1}+2 \epsilon(n+1) C_{3.1} \lambda^{n} \\
\leqslant & C_{3.1}(1 / 4+(n+1)(2 \delta+\epsilon)) \lambda^{n+1}+C_{\delta} \lambda^{n^{\frac{n^{2}+2 n}{n+1}}} .
\end{aligned}
$$


Choosing $\delta$ small enough to have $(n+1)\left(2 \delta+\epsilon_{0}\right) \leqslant 1 / 2$, for instance $\delta=1 /(16(n+1))$, we conclude that $\lambda \leqslant \lambda_{0}$, where $\lambda_{0}=\left(4 C_{\delta} / C_{3.1}\right)^{n+1}$. Therefore, in conclusion, considering both cases, for

$$
L_{0}=L_{0}(n)=1+\max \left\{D_{0}, \lambda_{0}, C_{3.1} / C_{2}\right\}
$$

we can deduce that $\left.f(l) \leqslant C_{3.1 .}\right]^{n+1}$ for every $l \in\left[L_{0}, L\right]$.

Finally, we indicate how to modify the previous arguments to prove Lemma 3.3 The basic point is that, now, we can use the uniform density distribution, which was proved as a consequence of Lemma 3.1

Proof of Lemma 3.3 The proof is similar to the previous one: from the uniform density distribution 2.21, proved using the $L^{\infty}$-estimate on $b$ in Proposition 3.2, for $l \geqslant d_{0}$, we always have

$$
\left|f_{Q_{l}} w\right| \leqslant|m|+\frac{2 n C_{3.2}}{d_{0}} \leqslant 1-\frac{1-|m|}{2}=\frac{1+|m|}{2} .
$$

Hence, applying (iii) ${ }^{\prime}$ of Lemma 4.2 in place of (iii), we see that, for every $l \geqslant d_{0}$,

$$
f(l) \leqslant 2 \delta l f^{\prime}(l)^{+}+2 G_{4.1]}(\delta)\left(f^{\prime}(l)^{+}\right)^{\frac{n+2}{n+1}}+C_{4.2]^{n}}+2 \epsilon f^{\prime}(l)^{+} .
$$

Reasoning as above, since the leading term is $l^{n}$, we can deduce the existence of $L_{0}$ such that $\left.f(l) \leqslant C_{3.3 .}\right]^{n}$ for $l \geqslant L_{0}$, proving the lemma.

\section{Scaling law and sharp interface limit}

In this section we show the last result of the work, namely Proposition 2.2. which, in terms of the parameter $\epsilon=\varepsilon^{2 / 3} \sigma^{1 / 3}$, reduces to proving the following proposition.

Proposition 5.1 There exists a constant $\gamma_{0}>0$ such that

$$
\lim _{\epsilon \rightarrow 0} \gamma_{\epsilon}=\gamma_{0}
$$

As pointed out in Section 2, this result allows us to recover the scaling law for the minimum energy (proved in [8] under more restrictive hypotheses): indeed, Proposition 5.1 and Theorem 3 imply that, in a subdomain of size of order $l \geqslant L_{0}$, the energy of minimizers of $G_{\epsilon}$ is nearly proportional to the volume of the domain, where the constant of proportionality $\gamma_{0}$ is the same for every $\epsilon$ small enough:

$$
\left|\min _{(u, b) \in \mathscr{B}_{L}} G_{\epsilon}\left(u, b, Q_{l}\right) / l^{n}-\gamma_{0}\right| \leqslant C(1 / l+\epsilon)+\left|\gamma_{\epsilon}-\gamma_{0}\right| \leqslant C(1 / l+\epsilon)+o(1) .
$$

Proposition 5.1 is a simple corollary of the $\Gamma$-convergence result of Modica and Mortola [23]. In order to state it, we recall some notation from Section 2 . We consider the functionals $E_{\epsilon}$ of 2.10 . defined in

$$
X_{m}=\left\{w \in L^{1}\left(\mathbb{T}_{L} ;[-1,1]\right): \int_{\mathbb{T}_{L}} w=m \in(-1,1)\right\} \subset L^{1}\left(\mathbb{T}_{L} ;[-1,1]\right)
$$


by

$$
E_{\epsilon}\left(w, \mathbb{T}_{L}\right)= \begin{cases}\int_{\mathbb{T}_{L}}\left(\epsilon|\nabla w|^{2}+\frac{W(w)}{\epsilon}+|\nabla \zeta|^{2}\right) & \text { if } w \in W^{1,2}\left(\mathbb{T}_{L} ;[-1,1]\right) \cap X_{m}, \\ +\infty & \text { if } w \in X_{m} \backslash W^{1,2}\left(\mathbb{T}_{L} ;[-1,1]\right),\end{cases}
$$

where $\zeta$ solves $-\Delta \zeta=w-m$. We denote by $E_{0}$ their sharp interface limit,

$$
E_{0}\left(w, \mathbb{T}_{L}\right)= \begin{cases}\int_{\mathbb{T}_{L}}\left(c_{W}|\nabla w|+|\nabla \zeta|^{2}\right) & \text { if } w \in B V\left(\mathbb{T}_{L} ;[-1,1]\right) \cap X_{m}, \\ +\infty & \text { if } w \in X_{m} \backslash B V\left(\mathbb{T}_{L} ;[-1,1]\right),\end{cases}
$$

with $\zeta$ as above and $c_{W}$ the usual constant depending on the potential $W$ (see [22] for the exact value). Then a simple consequence of the Modica-Mortola result is the following proposition (see also [29]).

Proposition 5.2 ( $\Gamma$-limit of $E_{\epsilon}$ ) For every $L>0$, we have $E_{\epsilon} \stackrel{\Gamma}{\rightarrow} E_{0}$ in $X_{m}$ as $\epsilon \rightarrow 0$, i.e. the following conclusions hold:

(a) (equicoercivity) every sequence $\left(w_{\epsilon}\right) \subset X_{m}$ such that $E_{\epsilon}\left(w_{\epsilon}\right) \leqslant C<+\infty$ is precompact in $L^{1}\left(\mathbb{T}_{L} ;[-1,1]\right)$, i.e. each subsequence $\left(w_{\epsilon_{n}}\right)$ has a converging sub-subsequence;

(b) (lim inf-inequality) for every $w_{\epsilon}, w \in X_{m}$ with $w_{\epsilon} \stackrel{L^{1}}{\rightarrow} w$, we have

$$
\liminf _{\epsilon \rightarrow 0} E_{\epsilon}\left(w_{\epsilon}, \mathbb{T}_{L}\right) \geqslant E_{0}(w)
$$

(c) (lim sup-inequality) for every $w \in X_{m}$ there exists a sequence $\left(w_{\epsilon}\right) \subset X_{m}$ such that $w_{\epsilon} \stackrel{L^{1}}{\rightarrow} w$ and

$$
\lim _{\epsilon \rightarrow 0} E_{\epsilon}\left(w_{\epsilon}, \mathbb{T}_{L}\right)=E_{0}(w) .
$$

In particular, the minimum values of the energy for the $E_{\epsilon}$ 's converge to the minimum value of $E_{0}$, $\gamma_{0}(L)=\min _{w \in L^{1}\left(\mathbb{T}_{L} ;[-1,1]\right)} E_{0}\left(w, \mathbb{T}_{L}\right)$, i.e.

$$
\gamma_{\epsilon}(L) \rightarrow \gamma_{0}(L) \quad \text { as } \epsilon \rightarrow 0 .
$$

Proof. The proof is immediate once one notices that $E_{\epsilon}$ is a continuous perturbation of the ModicaMortola functional $M_{\epsilon}$. Indeed, $u_{\epsilon} \stackrel{L^{1}}{\rightarrow} u$ implies $\nabla \zeta_{\epsilon} \stackrel{L^{2}}{\rightarrow} \nabla \zeta$ :

$$
\int_{\mathbb{T}_{L}}\left|\nabla \zeta_{\epsilon}-\nabla \zeta\right|^{2} \leqslant C \int_{\mathbb{T}_{L}}\left|u_{\epsilon}-u\right|^{2} \leqslant 2 C \int_{\mathbb{T}_{L}}\left|u_{\varepsilon}-u\right| \rightarrow 0
$$

Now, we are ready to prove Proposition 5.1

Proof of Proposition 5.1. We show that $\gamma_{\epsilon}$ is a Cauchy sequence in $\epsilon$ : for every $\delta>0$, we find $\theta>0$ such that

$$
\left|\gamma_{\epsilon^{\prime}}-\gamma_{\epsilon^{\prime \prime}}\right| \leqslant \delta \quad \forall \epsilon^{\prime}, \epsilon^{\prime \prime} \leqslant \theta .
$$


To show this, we choose $l$ such that $C_{[3.7} l \leqslant \delta / 4$. Hence, for $\epsilon^{\prime}, \epsilon^{\prime \prime} \leqslant \epsilon_{0}$, we have

$$
\begin{aligned}
& \left|\gamma_{\epsilon^{\prime}}-\gamma_{\epsilon^{\prime \prime}}\right| \leqslant\left|\gamma_{\epsilon^{\prime}}-\gamma_{\epsilon^{\prime}}(l)\right|+\left|\gamma_{\epsilon^{\prime}}(l)-\gamma_{\epsilon^{\prime \prime}}(l)\right|+\left|\gamma_{\epsilon^{\prime \prime}}(l)-\gamma_{\epsilon^{\prime \prime}}\right| \\
& \stackrel{3.30}{\leqslant} C_{3.7}\left(2 / l+\epsilon^{\prime}+\epsilon^{\prime \prime}\right)+\left|\gamma_{\epsilon^{\prime}}(l)-\gamma_{\epsilon^{\prime \prime}}(l)\right| \\
& \leqslant \delta / 2+C_{[3.7}\left(\epsilon^{\prime}+\epsilon^{\prime \prime}\right)+\left|\gamma_{\epsilon^{\prime}}(l)-\gamma_{0}\right|+\left|\gamma_{0}-\gamma_{\epsilon^{\prime \prime}}(l)\right| \text {. }
\end{aligned}
$$

Now, we take $\theta$ small enough to have $2 C_{3.7} 9 \leqslant \delta / 4$ and $\left|\gamma_{\epsilon}(l)-\gamma_{0}(l)\right| \leqslant \delta / 8$ for every $\epsilon \leqslant \theta$ (notice that this can always be achieved by Proposition 5.2). It is easy to notice that this choice of $\theta$ and (5.6) imply $(5.5)$, thus finishing the proof.

\section{Appendix}

Lemma 6.1 Assume $w \in W^{1,2}\left(Q_{l} ;[-1,1]\right)$ and $b \in L^{2}\left(Q_{l} ; \mathbb{R}^{n}\right)$ satisfy $\operatorname{div} b=w-m$ with $m \in(0,1)$. Assume that $l \geqslant 2$ and

$$
\left|f_{Q_{l}} w\right|=\left|m+\frac{1}{l^{n}} \int_{\partial Q_{l}} b \cdot v\right| \geqslant 1-\frac{1}{\sqrt{l}} .
$$

Then there exist constants $q_{6.1}$ and $R_{0}$ such that, for every $l \geqslant R_{0}$,

$$
\left.G_{\epsilon}\left(w, b, Q_{l}\right) \geqslant G_{6.1}\right]^{n+2} .
$$

Proof. We estimate the total energy with its nonlocal term, which, in turn, by a simple computation, can be estimated as follows:

$$
G_{\epsilon}\left(w, b, Q_{l}\right) \geqslant \int_{Q_{l}}|b|^{2} \geqslant \int_{B_{l / 2}}|b|^{2} \geqslant \int_{B_{l / 2}}|\nabla v|^{2},
$$

where $v$ solves the following equation in the ball $B_{l / 2}$ :

$$
\begin{cases}-\Delta v=w-m & \text { in } B_{l / 2} \\ v=0 & \text { on } \partial B_{l / 2}\end{cases}
$$

Without loss of generality, let $\int w>0$ and set

$$
A:=\left\{x \in Q_{l} \mid w(x) \leqslant(1+m) / 2=: \alpha\right\} .
$$

Clearly, we have

$$
\alpha|A|+\left(l^{n}-|A|\right) \geqslant \int_{Q_{l}} w \geqslant l^{n}(1-C / \sqrt{l}),
$$

from which we infer that $|A| \leqslant C l^{n-1 / 2}$. We define

$$
\varphi_{1}=\left\{\begin{array}{ll}
w-m & \text { in } Q_{l} \backslash A, \\
\alpha-m & \text { in } A,
\end{array} \quad \text { and } \quad \varphi_{2}=w-m-\varphi_{1}=(w-\alpha) \chi_{A},\right.
$$


and note that

$$
0<\alpha-m \leqslant \varphi_{1} \leqslant 1-m \text { and } \varphi_{2} \leqslant 0 .
$$

Hence, $v=v_{1}+v_{2}$, with

$$
\left\{\begin{array}{ll}
-\Delta v_{i}=\varphi_{i} & \text { in } B_{l / 2}, \\
v_{i}=0 & \text { on } \partial B_{l / 2},
\end{array} \quad \text { for } i=1,2,\right.
$$

and, from 6.5), we infer that $v_{2} \leqslant 0 \leqslant v_{1}$, as these are, respectively, superharmonic and subharmonic functions. So, the nonlocal term of the energy can be estimated in the following way:

$$
\begin{aligned}
\int_{B_{l / 2}}|\nabla v|^{2} & =-\int_{B_{l / 2}} v \Delta v=\int_{B_{l / 2}}(w-m) v_{1}+\int_{B_{l / 2}}(w-m) v_{2} \\
& \geqslant(\alpha-m) \int_{B_{l / 2} \backslash A} v_{1}-2 \int_{B_{l / 2} \cap A} v_{1}+2 \int_{B_{l / 2}} v_{2} .
\end{aligned}
$$

Now, using the maximum principle, since $\alpha-m \leqslant \varphi_{1} \leqslant 1-m$, we deduce that

$$
\frac{\alpha-m}{2 n} \eta(x) \leqslant v_{1}(x) \leqslant \frac{1-m}{2 n} \eta(x),
$$

where $\eta(x)=l^{2} / 4-|x|^{2}$. Hence, from $\int_{B_{l / 2} \cap A} \eta \leqslant C l^{2}|A| \leqslant C l^{n+3 / 2}$, we easily infer

$$
\begin{gathered}
(\alpha-m) \int_{B_{l / 2} \backslash A} v_{1} \geqslant C \int_{B_{l / 2} \backslash A} \eta \geqslant C l^{n+2}-C l^{n+3 / 2}, \\
-2 \int_{B_{l / 2} \cap A} v_{1} \geqslant-C \int_{B_{l / 2} \cap A} \eta \geqslant-C l^{n+3 / 2} .
\end{gathered}
$$

On the other hand, for what concerns $v_{2}$, using the $L^{2}$-estimate for the Laplace equation, we directly conclude that

$$
-\int_{B_{l / 2}} v_{2} \leqslant\left\|v_{2}\right\|_{L^{2}}\left|B_{l / 2}\right|^{1 / 2} \leqslant C l^{2}\left\|\varphi_{2}\right\|_{L^{2}} l^{n / 2} \leqslant C|A|^{1 / 2} l^{n / 2+2} \leqslant C l^{n+7 / 4} .
$$

Therefore, collecting 6.6- 6.8), we deduce the claim, i.e. we find constants $G_{6.1}$ and $R_{0}$ such that, for $l \geqslant R_{0}$,

$$
\begin{aligned}
E_{\varepsilon}\left(w, b, Q_{l}\right) & \geqslant \int_{B_{l / 2}}|\nabla v|^{2} \geqslant(\alpha-m) \int_{B_{l / 2} \backslash A} v_{1}-2 \int_{B_{l / 2} \cap A} v_{1}+2 \int_{B_{l / 2}} v_{2} \\
& \geqslant C\left(l^{n+2}-l^{n+3 / 2}-l^{n+7 / 4}\right) \geqslant C{ }_{6.1}^{l+2} .
\end{aligned}
$$

\section{REFERENCES}

1. Alberti, G., Choksi, R., \& Otтo, F. Uniform energy distribution for an isoperimetric problem with long-range interactions. J. Amer. Math. Soc. 22 (2009), 569-605. MR 2476783

2. Alberti, G., \& MÜLler, S. A new approach to variational problems with multiple scales. Comm. Pure Appl. Math. 54 (2001), 761-825. Zbl 1021.49012 MR 1823420 
3. Bahiana, T., \& Oono, Y. Cell dynamical system approach to block copolymers. Phys. Rev. A 41 (1990), 6763-6771.

4. Bates, F., \& Fredrickson, G. Block copolymers-designer soft materials. Physics Today 52-4 (1999), 32-38.

5. BRAides, A. $\Gamma$-convergence for Beginners. Oxford Lecture Ser. Math. Appl. 22, Oxford Univ. Press, Oxford (2002). Zbl pre01865939 MR 1968440

6. Chen, X., \& Oshita, Y. Periodicity and uniqueness of global minimizers of an energy functional containing a long-range interaction. SIAM J. Math. Anal. 37 (2005), 1299-1332. Zbl 1114.49002 MR 2192296

7. Chen, X., \& Oshita, Y. An application of the modular function in nonlocal variational problems. Arch. Ration. Mech. Anal. 186 (2007), 109-132. Zbl 1147.74024 MR 2338353

8. Choksi, R. Scaling laws in microphase separation of diblock copolymers. J. Nonlinear Sci. 11 (2001), 223-236. Zbl 1023.82015 MR 1852942

9. Choksi, R., \& V, R. Kohn. Bounds on the micromagnetic energy of a uniaxial ferromagnet. Comm. Pure Appl. Math. 51 (1998), 259-289. zbl 0909.49004 MR 1488515

10. Chокsi, R., Kohn, R. V., \& Отто, F. Domain branching in uniaxial ferromagnets: a scaling law for the minimum energy. Comm. Math. Phys. 201 (1999), 61-79. Zbl 1023.82011 MR 1669433

11. CHOKSI, R., \& REN, X. On the derivation of a density functional theory for microphase separation of diblock copolymers. J. Statist. Phys. 113 (2003), 151-176. Zbl 1034.82037 MR 2012976

12. ChOKsi, R., \& REN, X. Diblock copolymer/homopolymer blends: derivation of a density functional theory. Phys. D 203 (2005), 100-119. Zbl 1120.82307 MR 2135136

13. Choksi, R., \& Sternberg, P. Periodic phase separation: the periodic Cahn-Hilliard and isoperimetric problems. Interfaces Free Bound. 8 (2006), 371-392. Zbl 1109.35092 MR 2273234

14. Choksi, R., \& Sternberg, P. On the first and second variations of a nonlocal isoperimetric problem. J. Reine Angew. Math. 611 (2007), 75-108. Zbl 1132.35029 MR 2360604

15. Conti, S. Branched microstructures: scaling and asymptotic self-similarity. Comm. Pure Appl. Math. $\mathbf{5 3}$ (2000), 1448-1474. Zbl 1032.74044 MR 1773416

16. Conti, S., DeSimone, A., Dolzmann, G., Müller, S., \& Otto, F. Multiscale modeling of materials - the role of analysis. In: Trends in Nonlinear Analysis, Springer, Berlin (2003), 375-408. Zbl 1065.74056 MR 1999106

17. Evans, L. C. Partial Differential Equations. Grad. Stud. Math. 18, Amer. Math. Soc., Providence, RI (1998). Zbl 0902.35002 MR 1625845

18. Gilbarg, D., \& Trudinger, N. S. Elliptic Partial Differential Equations of Second Order. Classics in Math., Springer, Berlin (2001). Reprint of the 1998 edition. Zbl 1042.35002 MR 1814364

19. Hamley, I. The Physics of Block Copolymers. Oxford Univ. Press, Oxford (1999).

20. KoHN, R. V. Energy-driven pattern formation. In: International Congress of Mathematicians. Vol. I, Eur. Math. Soc., Zürich (2007), 359-383. Zbl 1140.49030 MR 2334197

21. Kohn, R. V., \& Müller, S. Surface energy and microstructure in coherent phase transitions. Comm. Pure Appl. Math. 47 (1994), 405-435. Zbl $0803.49007 \mid$ MR 1272383

22. ModicA, L. The gradient theory of phase transitions and the minimal interface criterion. Arch. Ration. Mech. Anal. 98 (1987), 123-142. Zbl 0616.76004 MR 0866718

23. Modica, L., \& Mortola, S. Un esempio di $\Gamma^{-}$-convergenza. Boll. Un. Mat. Ital. B (5) 14 (1977), 285-299. Zbl 0356.49008 MR 0445362

24. MÜLLER, S. Singular perturbations as a selection criterion for periodic minimizing sequences. Calc. Var. Partial Differential Equations 1 (1993), 169-204. Zbl $0821.49015 \mid$ MR 1261722

25. Muratov, C. B. Theory of domain patterns in systems with long-range interactions of Coulomb type. Phys. Rev. E (3) 66 (2002), 066108, 25 pp. MR 1953930 
26. NishiurA, Y., \& OHNishi, I. Some mathematical aspects of the micro-phase separation in diblock copolymers. Phys. D 84 (1995), 31-39. MR 1334695

27. Ohnishi, I., Nishiura, Y., Imai, M., \& Matsushita, Y. Analytical solutions describing the phase separation driven by a free energy functional containing a long-range interaction term. Chaos 9 (1999), 329-341. Zbl 0970.35151 MR 1697656

28. OHTA, T., \& KAWASAKI, K. Equilibrium morphology of diblock copolymer melts. Macromolecules 19 (1986), 2621-2632.

29. REN, X., \& WEI, J. On the multiplicity of solutions of two nonlocal variational problems. SIAM J. Math. Anal. 31 (2000), 909-924. Zbl 0973.49007 MR 1752422

30. REN, X., \& WEI, J. Concentrically layered energy equilibria of the di-block copolymer problem. Eur. J. Appl. Math. 13 (2002), 479-496. Zbl 1010.82041 MR 1939157

31. REN, X., \& WEI, J. On energy minimizers of the diblock copolymer problem. Interfaces Free Bound. 5 (2003), 193-238. Zbl 1031.49035 MR 1980472

32. REN, X., \& WEI, J. On the spectra of three-dimensional lamellar solutions of the diblock copolymer problem. SIAM J. Math. Anal. 35 (2003), 1-32. Zbl 1055.35041 MR 2001463

33. REN, X., \& WEI, J. Wriggled lamellar solutions and their stability in the diblock copolymer problem. SIAM J. Math. Anal. 37 (2005), 455-489. Zbl 1136.35372 MR 2176111

34. REN, X., \& WEI, J. Droplet solutions in the diblock copolymer problem with skewed monomer composition. Calc. Var. Partial Differential Equations 25 (2006), 333-359. Zbl 1088.82033 MR 2201676

35. REN, X., \& WEI, J. Existence and stability of spherically layered solutions of the diblock copolymer equation. SIAM J. Appl. Math. 66 (2006), 1080-1099. Zbl 1103.34046 MR 2216732

36. REN, X., \& WeI, J. Many droplet pattern in the cylindrical phase of diblock copolymer morphology. Rev. Math. Phys. 19 (2007), 879-921. Zbl 1145.82007 MR 2349026

37. REN, X., \& WEI, J. Spherical solutions to a nonlocal free boundary problem from diblock copolymer morphology. SIAM J. Math. Anal. 39 (2008), 1497-1535. Zbl 1153.35091 MR 2377287

38. Reshetnyak, Y. G. Sobolev-type classes of mappings with values in metric spaces. In: The Interaction of Analysis and Geometry, Contemp. Math. 424, Amer. Math. Soc., Providence, RI (2007), 209-226. Zbl 1153.46022 MR 2316339

39. Ros, A. Stable periodic constant mean curvature surfaces and mesoscopic phase separation. Interfaces Free Bound. 9 (2007), 355-365. Zbl 1142.53013 MR 2341847

40. SpadAro, E. N. A variational model for periodic pattern formation. Master thesis, available at http://etd.adm.unipi.it/theses/available/etd-09142006-181514/, 2006 (in Italian).

41. Teramoto, T., \& Nishiura, Y. Double gyroid morphology in a gradient system with non-local effects. J. Phys. Soc. Japan 71 (2002), 1611-1614.

42. Thomas, E. L., Anderson, D. M., Henkee, C. S., \& Hoffman, D. Periodic area-minimizing surfaces in diblock copolymers. Nature 334 (1988), 598-601.

43. YIP, N. K. Structure of stable solutions of a one-dimensional variational problem. ESAIM Control Optim. Calc. Var. 12 (2006), 721-751. Zbl 1117.49025 MR 2266815 